\title{
ApoE4 markedly exacerbates tau-mediated neurodegeneration in a mouse model of tauopathy
}

Yang Shi ${ }^{1}$, Kaoru Yamada ${ }^{2}$, Shane Antony Liddelow ${ }^{3,4}$, Scott T. Smith ${ }^{5}$, Lingzhi Zhao ${ }^{6}$, Wenjie Luo ${ }^{6}$, Richard M. Tsai ${ }^{7}$, Salvatore Spina ${ }^{7}$, Lea T. Grinberg ${ }^{7,8}$, Julio C. Rojas ${ }^{7}$, Gilbert Gallardo ${ }^{1}$, Kairuo Wang ${ }^{1}$, Joseph Roh ${ }^{1}$, Grace Robinson ${ }^{9}$, Mary Beth Finn ${ }^{1}$, Hong Jiang ${ }^{1}$, Patrick M. Sullivan ${ }^{10}$, Caroline Baufeld ${ }^{5}$, Michael W. Wood ${ }^{11}$, Courtney Sutphen ${ }^{1}$, Lena McCue ${ }^{12}$, Chengjie Xiong $^{12}$, Jorge L. Del-Aguila ${ }^{13}$, John C. Morris ${ }^{1}$, Carlos Cruchaga ${ }^{13,14}$, Alzheimer's Disease Neuroimaging Initiative*, Anne M. Fagan ${ }^{1}$, Bruce L. Miller ${ }^{7}$, Adam L. Boxer ${ }^{7}$, William W. Seeley ${ }^{7,8}$, Oleg Butovsky ${ }^{5,15}$, Ben A. Barres ${ }^{3}$, Steven M. Paul ${ }^{16}$ \& David M. Holtzman ${ }^{1}$

APOE4 is the strongest genetic risk factor for late-onset Alzheimer disease. ApoE4 increases brain amyloid- $\beta$ pathology relative to other ApoE isoforms ${ }^{1}$. However, whether $A P O E$ independently influences tau pathology, the other major proteinopathy of Alzheimer disease and other tauopathies, or tau-mediated neurodegeneration, is not clear. By generating P301S tau transgenic mice on either a human ApoE knock-in (KI) or ApoE knockout (KO) background, here we show that P301S/E4 mice have significantly higher tau levels in the brain and a greater extent of somatodendritic tau redistribution by three months of age compared with P301S/E2, P301S/E3, and $\mathrm{P} 301 \mathrm{~S} / \mathrm{EKO}$ mice. By nine months of age, P301S mice with different ApoE genotypes display distinct phosphorylated tau protein ( $p$-tau) staining patterns. P301S/E4 mice develop markedly more brain atrophy and neuroinflammation than P301S/E2 and P301S/E3 mice, whereas P301S/EKO mice are largely protected from these changes. In vitro, E4-expressing microglia exhibit higher innate immune reactivity after lipopolysaccharide treatment. Co-culturing P301S tau-expressing neurons with E4-expressing mixed glia results in a significantly higher level of tumour-necrosis factor- $\alpha$ (TNF- $\alpha$ ) secretion and markedly reduced neuronal viability compared with neuron/E2 and neuron/E3 co-cultures. Neurons co-cultured with EKO glia showed the greatest viability with the lowest level of secreted TNF- $\alpha$. Treatment of P301S neurons with recombinant ApoE (E2, E3, E4) also leads to some neuronal damage and death compared with the absence of ApoE, with ApoE4 exacerbating the effect. In individuals with a sporadic primary tauopathy, the presence of an $\varepsilon 4$ allele is associated with more severe regional neurodegeneration. In individuals who are positive for amyloid- $\beta$ pathology with symptomatic Alzheimer disease who usually have tau pathology, $\varepsilon 4$-carriers demonstrate greater rates of disease progression. Our results demonstrate that ApoE affects tau pathogenesis, neuroinflammation, and tau-mediated neurodegeneration independently of amyloid- $\beta$ pathology. ApoE4 exerts a 'toxic' gain of function whereas the absence of ApoE is protective.

In 1993, APOE4 was identified as a strong genetic risk factor for late-onset Alzheimer disease ${ }^{2}$. Subsequently, a large amount of evidence
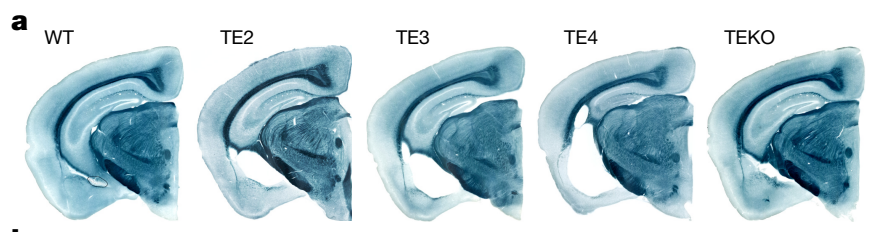

b
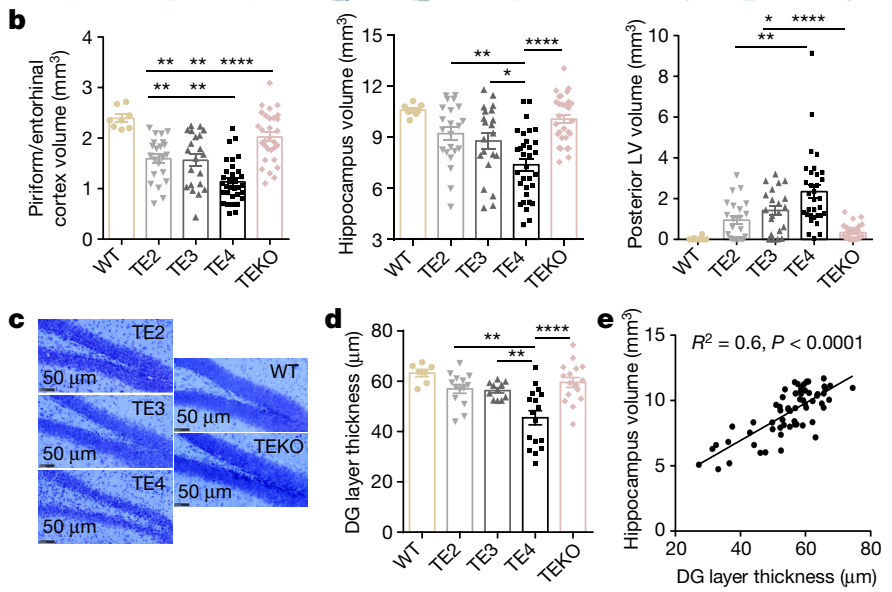

Figure 1 | ApoE4 exacerbates neurodegeneration in P301S mice whereas genetic ablation of ApoE is associated with less damage. a, Representative images of 9-month-old WT and TE mouse brain sections stained with Sudan black. b, Volumes of the piriform/entorhinal cortex, hippocampus, and posterior lateral ventricle (LV) in 9- to 10-month-old WT and TE mice (WT, $n=8$; TE2, $n=22$; TE3, $n=21$; TE4, $n=32$; TEKO, $n=30$ ). c, $\mathbf{d}$, Thickness of the granule cell layer of the dentate gyrus (DG) in 9-month-old WT and TE mice with cresyl violet staining (WT, $n=7$; TE2, $n=14$; TE3, $n=11$; TE4, $n=17$; TEKO, $n=16$ ). e, Correlation between granule layer thickness and hippocampal volume, $n=62$ biologically independent animals. Pearson correlation analysis, $R^{2}=0.6, P<0.0001$ (two-sided). Data expressed as mean \pm s.e.m. One-way analysis of variance (ANOVA) with Tukey's post hoc test (two-sided) was used for all statistical analyses except Kruskal-Wallis test with Dunn's multiple comparisons test (two-sided) was performed for analysis of posterior lateral ventricle volume. $* P<0.05, * * P<0.01, * * * P<0.001, * * * * P<0.0001$.

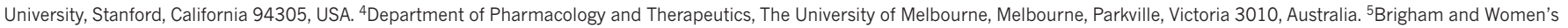

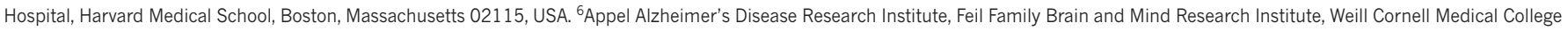
of Cornell University, New York, New York 10021, USA. ${ }^{7}$ Memory and Aging Center, Department of Neurology, University of California, San Francisco, California 94143 USA. ${ }^{8}$ Department of

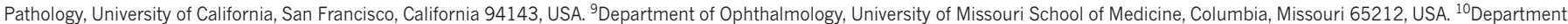
of Medicine, Duke University Medical Center, Durham Veterans Health Administration Medical Center's Geriatric Research, Education and Clinical Center, Durham, North Carolina 27705, USA. ${ }^{11}$ AstraZeneca R\&D, Wilmington, Delaware 19850, USA. ${ }^{12}$ Division of Biostatistics, Washington University in St Louis, St Louis, Missouri 63110 , USA. ${ }^{13}$ Department of Psychiatry, Washington University School of Medicine, 660 S. Euclid Avenue B8134, St. Louis, Missouri 63110, USA. ${ }^{14}$ Department of Developmental Biology, Washington University School of Medicine, 660 S. Euclid

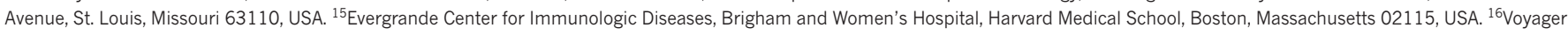
Therapeutics, Cambridge, Massachusetts 02139, USA.

* Lists of participants and their affiliations appear in the Supplementary Information. 
has demonstrated that a major mechanism by which ApoE influences Alzheimer disease is via ApoE influencing amyloid- $\beta$ deposition in both a dose and isoform-specific fashion (E4 > E3 $>$ E2) $)^{1}$. However, there are probably other mechanisms by which ApoE influences not only Alzheimer disease but also other neurodegenerative diseases. A large body of evidence demonstrates that although amyloid- $\beta$ is probably a key initiator in Alzheimer disease pathogenesis, its aggregation and accumulation poorly correlate with disease symptoms or tissue $\operatorname{loss}^{3,4}$. In contrast, accumulation of tau in brains with Alzheimer disease and in primary tauopathies strongly correlates with clinical signs and neurodegeneration ${ }^{3-5}$. However, so far there is only circumstantial evidence that ApoE influences tauopathy independently of amyloid- $\beta$. ApoE has been shown to directly bind tau in vitro ${ }^{6}$, and neuronal expression of human ApoE in vivo results in tau hyperphosphorylation $(\mathrm{E} 4>\mathrm{E} 3)^{7}$. Recent genome-wide association studies show a strong and significant association of $A P O E$ with cerebrospinal fluid (CSF) tau and p-tau after correcting for the effect of $A P O E$ on amyloid- $\beta 42$ levels ${ }^{8}$. In patients with frontotemporal dementia, a large percentage of whom have tauopathy, $\varepsilon 4$ allele frequency was reported to be significantly elevated ${ }^{9,10}$, and $\varepsilon 4$ carriers have greater atrophy in affected brain regions ${ }^{11}$ as well as exacerbated behavioural deficits ${ }^{12}$. These data suggest that ApoE may directly influence tau pathology and tau-mediated neurodegeneration.

To determine whether the presence of ApoE or human ApoE isoforms affect tau pathology and tau-related neuropathology, we used a P301S tauopathy mouse model, which overexpresses 1N4R human tau containing the P301S mutation that causes a form of frontotemporal dementia ${ }^{13}$. We generated P301S mice on either a human ApoE KI or ApoE KO background, designated as TE (Tau/ApoE) mice. We observed significantly more brain atrophy in 9-month-old P301S/E4 (TE4) mice compared with P301S/E2 (TE2) and P301S/E3 (TE3) mice (Fig. 1a, b), but no change in 3-month-old TE mice or 9-month-old non-tau transgenic ApoE KI mice (Extended Data Fig. 1). The atrophy primarily occurred in the hippocampus, piriform/entorhinal cortex, and amygdala, and was accompanied by significant lateral ventricular enlargement (Fig. 1a, b). The granule cell layer in the dentate gyrus (Fig. 1c, d) and the pyramidal cell layer in the CA1 region (Extended Data Fig. 2) were noticeably and significantly thinner in TE4 mice, and the thickness correlated highly with hippocampal volume (Fig. 1e). Notably, the absence of ApoE in P301S mice (TEKO) largely attenuated neuronal loss and brain atrophy observed in P301S mice expressing human ApoE, and almost completely abolished ventricular dilatation (Fig. 1a-d). These results revealed an important role of ApoE in regulating tau-mediated neurodegeneration, with ApoE4 causing more severe damage and the absence of ApoE being protective.

Human tau levels in brain lysates were analysed by quantitative ELISA after sequential biochemical extraction in RAB (salt buffer), RIPA (detergent buffer), and 70\% formic acid, which contain soluble tau, less soluble tau, and highly insoluble tau, respectively. At three months of age, before onset of overt tau pathology, TE4 mice had significantly higher tau levels in the RAB fraction than other ApoE genotypes (Fig. 2a). At nine months of age, when large amounts of tau pathology had developed, TE4 mice still had significantly higher tau levels, but the elevated tau had changed from being in the RAB to the RIPA fraction (Fig. 2a), indicating a greater amount of tau in a more insoluble fraction. The higher tau accumulation in TE4 mice was not due to differences in tau synthesis (Extended Data Fig. 3a). We found significant changes of autophagy-related gene expression in 9-month-old TE4 mice and 9-month-old non-tau transgenic ApoE4 KI mice (Extended Data Fig. 3b, c), suggesting ApoE4 may affect autophagy-mediated tau clearance. This is consistent with a previous report of impaired autophagy associated with ApoE $4^{14}$. ApoE levels in the TE mice followed the pattern E2 $>$ E3 $>$ E4 (Extended Data Fig. 3d, e), consistent with what has been reported in ApoE KI mice ${ }^{15}$. No major alteration of ApoE levels was observed in the presence of tau pathology. Hyperphosphorylated tau, identified by p-tau staining with the AT8
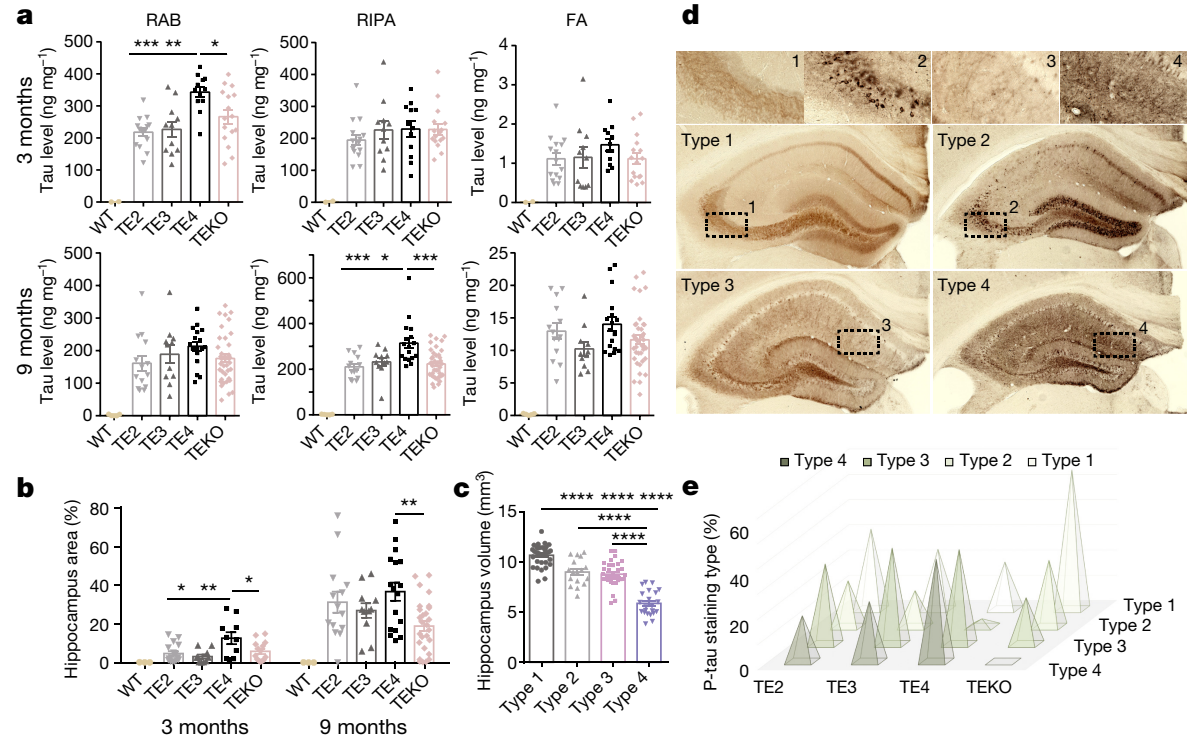

Figure 2 | ApoE genotypes differentially regulate tau pathology. a, Human P301S tau levels in TE mice were measured by ELISA in RAB, RIPA, and $70 \%$ formic acid fractions respectively at two time points: 3 months (WT, $n=2$; TE2, $n=15$; TE3, $n=11$; TE4, $n=12$; TEKO, $n=16$ ) and 9 months (WT, $n=5$; TE2, $n=14$; TE3, $n=11$; TE4, $n=17$; TEKO, $n=38$ ). $\mathbf{b}$, P-tau (AT8) covered area in 3- and 9-month-old TE mouse hippocampus. c, P-tau staining patterns were associated with different degrees of brain atrophy, $n=104$ biologically independent animals. a-c, Data expressed as mean \pm s.e.m., one-way ANOVA with Tukey's post hoc test (two-sided). d, Four distinct p-tau staining patterns were identified on the basis of hippocampal staining features. Type 1 has intense mossy fibre staining as well as diffuse cell body staining in the dentate gyrus granule cell layer and CA1 pyramidal cell layer; type 2 has compact and dense tangle-like cell body staining primarily in the dentate gyrus granule cells and CA3 pyramidal cells, but also has sparse staining in the CA1 region; type 3 has staining primarily in the neuropil of the stratum radiatum of the CA region with clear staining of dendrites from pyramidal neurons and only some staining in the neuronal cell bodies; type 4 has dense staining over the entire hippocampus, unlike other staining patterns; type 4 staining is fragmented, dotted, and grainy. e, Distribution of the four p-tau staining types in 9- to 10-month-old TE mice (TE2, $n=22$; TE3, $n=21$; TE4, $n=32$; TEKO, $n=38$ ). Fisher's exact test; two-sided (all groups, $P=3.4 \times 10^{-5}$; TE2 versus TEKO, $P=0.021$; TE3 versus TEKO, $P=0.0016$; TE4 versus TEKO, $\left.P=1.9 \times 10^{-7}\right) . * P<0.05$, $* * P<0.01, * * * P<0.001, * * * * P<0.0001$. 


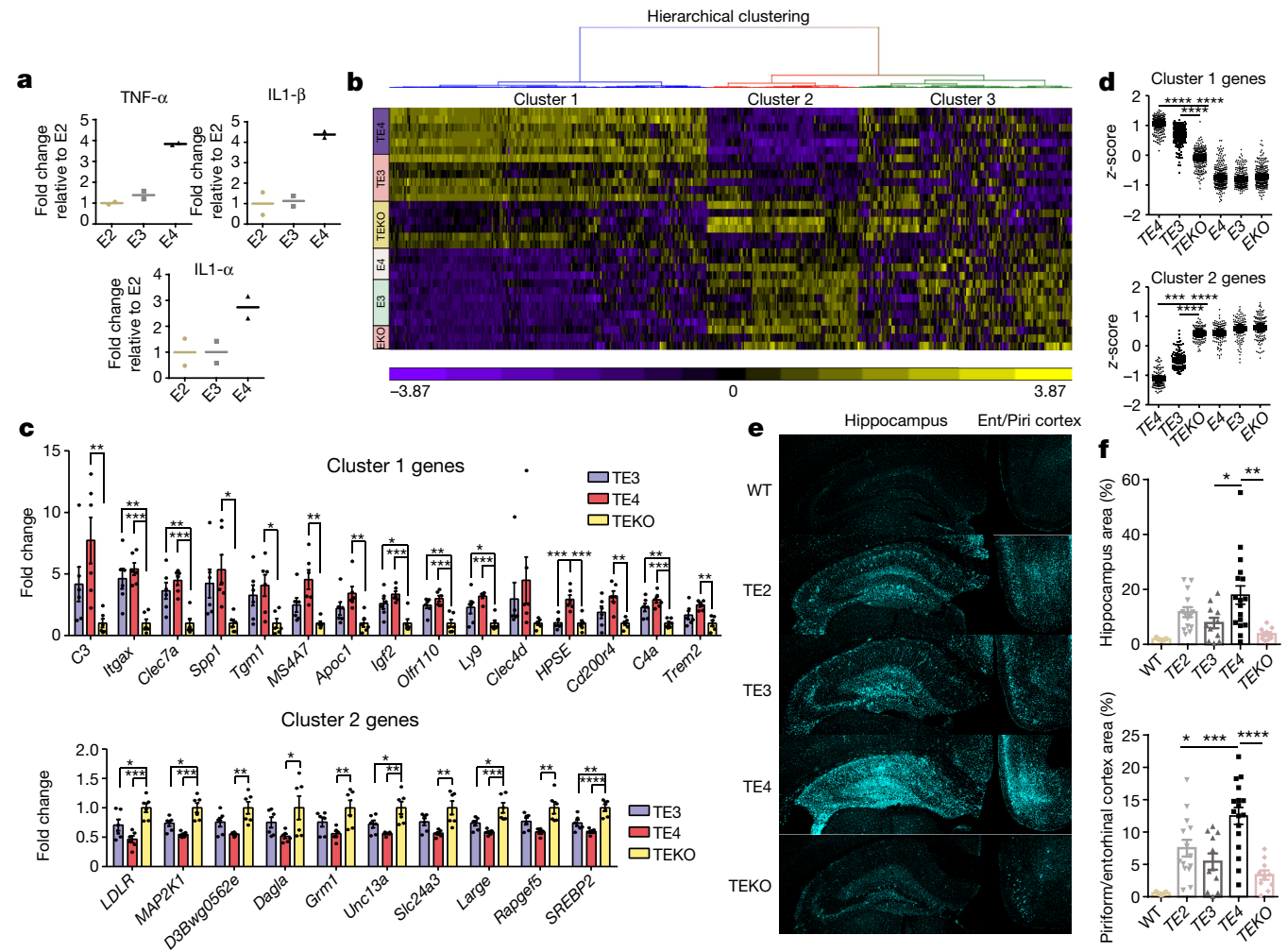

Figure 3 | ApoE strongly modulates microglial activation. a, Cultured microglia (E2, E3, E4) derived from human ApoE KI mouse brains were treated with $1 \mathrm{ng} \mathrm{ml}^{-1}$ LPS for $24 \mathrm{~h}$, cytokine levels in the medium were measured by ELISA (two wells per genotype), experiment replicated three times. b, Nanostring analysis for microglial gene expression in 9-month-old TE or EKI/EKO mouse hippocampus. Heatmap generated by hierarchical gene clustering on the basis of genotypes (horizontal, 534 microglial genes; vertical, individual mouse samples; TE3, TE4, TEKO, E3, $n=6$; E4, $n=4$; EKO, $n=3$ ). c, Top differentially expressed cluster 1 and cluster 2 genes from the heatmap (criteria: fold change TE4 versus TEKO high to low, $P<0.01$; fold change TE4 versus TE3 $>1.2$ ). Cluster 1 :

antibody, revealed a greater p-tau-covered area in 3-month-old TE4 mouse hippocampus (Fig. 2b).

Although the pathological p-tau signal first appeared in the mossy fibres, axons of dentate gyrus granule cells in the hippocampus, TE4 mice also showed more intense dentate gyrus cell body staining (Extended Data Fig. 4), indicating a greater redistribution of pathological p-tau from axons to cell bodies at an early age. At nine months of age, the differences in overall $\mathrm{p}$-tau immunoreactivity between TE mice decreased (Fig. 2b), with the emergence of four major p-tau staining patterns, designated as types 1-4 (Fig. 2d). These staining patterns strongly correlated with the level of brain atrophy, with type 1 associated with the most preserved brain and type 4 associated with the greatest atrophy (Fig. 2c), suggesting potential differential toxicity associated with different $\mathrm{p}$-tau immunoreactivity patterns. The distribution of p-tau immunoreactivity patterns differed across ApoE genotypes. Type 1 and type 2 were enriched in TEKO mice whereas type 4 was enriched in TE4 mice (Fig. 2e). The featured distribution of these p-tau immunoreactivity patterns, which either represent different tau structures or progressively more advanced pathological tau stages, indicate ApoE affects either tau conformation or tau pathology progression.

While pathological tau may directly cause neurodegeneration, it, along with degenerating neurons, may also induce chronic neuroinflammation, which can lead to neuronal death via various mechanisms ${ }^{16,17}$. Previous studies have shown that ApoE4 is associated with higher innate immune reactivity than ApoE2 and ApoE3 (refs 18, 19). We confirmed this result in cultured microglia with lipopolysaccharide (LPS) stimulation (Fig. 3a). This inherently higher inflammatory reactivity associated proinflammatory genes; cluster 2: cellular function-related genes (metabolism, signalling, transcription, etc.); cluster 3: homeostatic genes/ genes below detection. d, $Z$-score of genes from cluster 1 or cluster 2 for all groups. Kruskal-Wallis test with Dunn's multiple comparisons test (two-sided) was performed for statistical analysis. e, f, CD68 (activated microglia) staining and quantification in 9-month-old TE mice (WT, $n=5$; TE2, $n=14$; TE3, $n=11$; TE4, $n=17$; TEKO, $n=10$ ). Ent/Piri, entorhinal and piriform. Data expressed as mean \pm s.e.m., one-way ANOVA with Tukey's post hoc test, two-sided. $* P<0.05$, $* * P<0.01, * * * P<0.001, * * * * P<0.0001$.

with ApoE4 may further exacerbate neurodegeneration. When we assessed microglial gene expression profiles in 9-month-old TE3, TE4, and TEKO mice as well as in 9-month-old non-tau transgenic ApoE $\mathrm{KI}$ and ApoE KO mice using a customized MG550 microglial gene chip (see Methods), we observed a marked upregulation of proinflammatory genes (cluster 1) and a concomitant downregulation of genes involved in normal cell function (cluster 2) in TE4 mice, whereas microglia in TEKO mice largely remained in a homeostatic state (Fig. 3b-d and Supplementary Information for pathway analysis and gene list). Consistent with this, CD68-positive microglial staining, which represents an activated state of microglia, was significantly elevated in TE4 mice, and was drastically reduced in TEKO mice (Fig. 3e, f). Notably, no proinflammatory gene activation and no or minimal gene expression differences were observed in 9-month-old ApoE KI and ApoE KO mice in the absence of P301S tau (Fig. 3b, d and Extended Data Fig. 5a, b) or in 3-month-old TE mice (Extended Data Fig. 5c, d), indicating the necessity of tau pathology to initiate microglial activation and to enable the ApoE genotype-dependent effect on microglial activation. Intriguingly, p-tau immunoreactivity patterns were highly associated with the microglial activation profile, with type 1 staining displaying the lowest, type 2 and type 3 intermediate, and type 4 the strongest microglial activation (Extended Data Fig. 6), suggesting potentially distinct intrinsic capacities of different p-tau patterns to induce neuroinflammation.

Another key cell type in the brain, astrocytes, also play an essential role in neuroinflammation. Recent studies have identified two distinct types of reactive astrocyte in mice, depending on the initiating injury: 'A1' induced by LPS-elicited inflammation, and 'A2' induced by ischaemia ${ }^{20}$. 


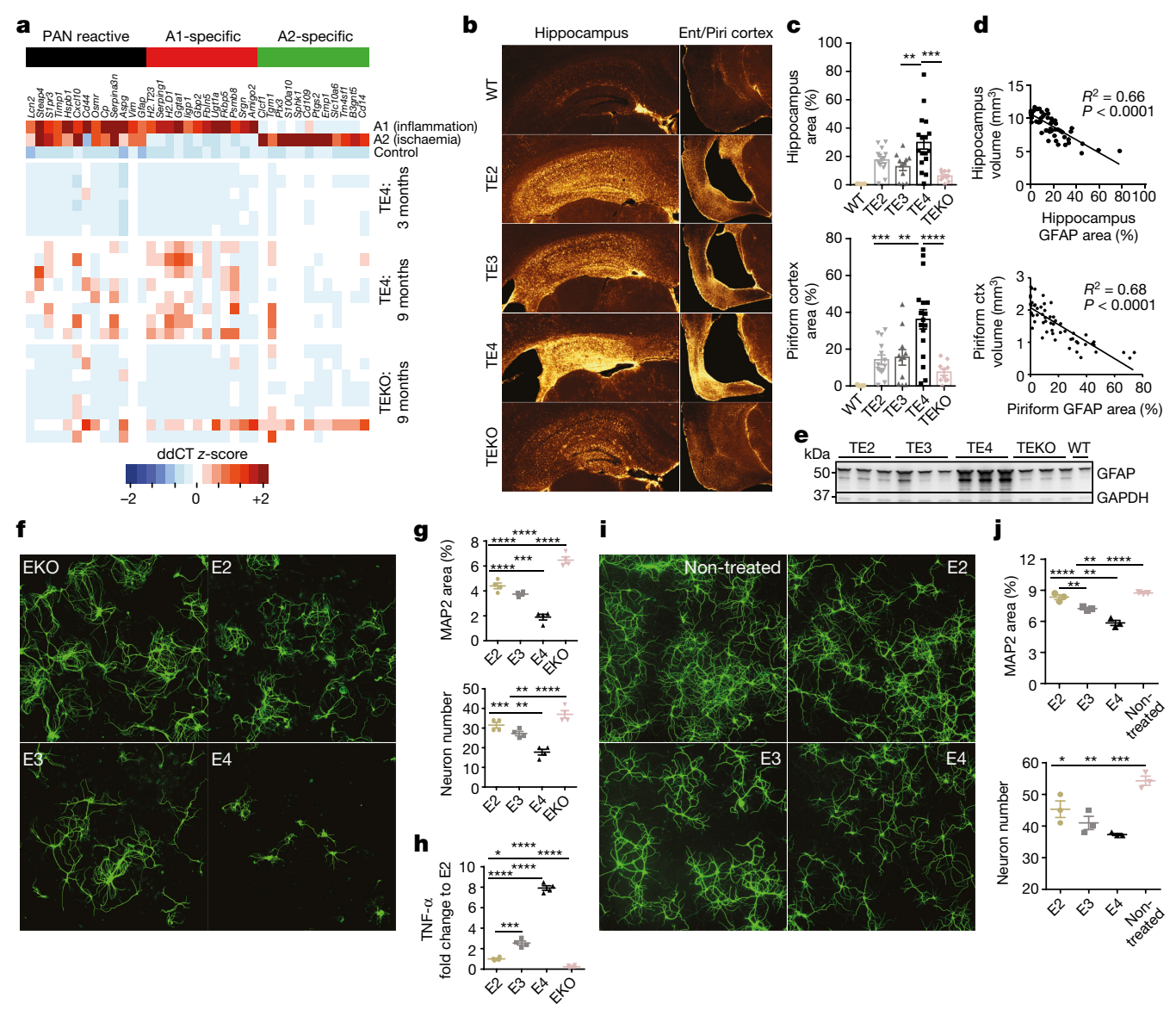

Figure 4 | ApoE4 leads to robust astrocytic activation and promotes neuronal death in vitro whereas the absence of ApoE results in less astrocytic activation and preserves neuronal integrity. a, Microfluidic quantitative PCR with reverse transcription (qRT-PCR) for activated astrocytic genes in 9-month-old TE4 $(n=8)$, TEKO $(n=8)$, and 3-monthold TE4 mice $(n=6)$. A1-specific: genes activated only by LPS; A2-specific: genes activated only by ischaemia; PAN reactive: genes activated by either LPS or ischaemia. b, GFAP staining in 9-month-old TE mice (WT, $n=5$; TE2, $n=14$; TE3, $n=11$; TE4 $n=17$; TEKO, $n=10)$. c, Quantification of area covered by GFAP immunoreactivity. d, Correlation between GFAP immunoreactivity and brain volume. $N=57$ biologically independent animals. Pearson correlation analysis (two-sided). Hippocampus: $R^{2}=0.66$, $P<0.0001$; piriform cortex: $R^{2}=0.68, P<0.0001$. e, Western blot for GFAP in 9-month-old TE mice $(n=3)$. For gel source data, see Supplementary Fig. 1.

These reactive astrocytes are defined by three cassettes of genes: A1-specifc (inflammation only), A2-specific (ischaemia only), and PAN reactive (upregulated in both injuries) ${ }^{20}$. Recent data show that inflammation-induced reactive microglia produce specific cytokines (TNF- $\alpha$, IL1- $\alpha, C 1 q$ ) that can activate 'A1' astrocytes, which lose many normal astrocytic functions, and secrete toxic factors able to rapidly induce neu-

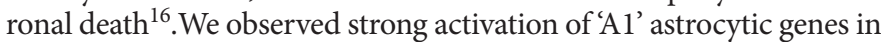
9-month-old TE4 mice, but not in 9-month-old TEKO mice or 3-monthold TE4 mice (Fig. 4a), nor in 9-month-old wild type (WT) and non-tau transgenic ApoE KI mice (Extended Data Fig. 7). In addition, glial fibrillary acidic protein (GFAP) signal was drastically elevated in 9-month-old TE4 mice, but not in TEKO mice (Fig. 4b, c, e). The amount of GFAP immunoreactivity highly correlated with brain volume (Fig. 4d), strongly suggesting a detrimental role of reactive astrocytes in neurodegeneration.

Remarkably, the detrimental effect of ApoE4 on neurodegeneration and the protective effect associated with the absence of ApoE were robustly recapitulated in vitro. When co-culturing P301S tau-expressing neurons with mixed glial cells derived from ApoE KI or ApoE KO mice, drastic neuronal death occurred in the neuron/E4 glia co-culture, whereas neurons co-cultured with EKO glia showed the least injury f, Representative images of primary WT neurons infected with AAV2/ 8-synapsin-P301S human tau co-cultured with mixed glia cells (80-90\% astrocytes, 10-20\% microglia) derived from human ApoE KI (E2, E3, E4) and ApoE KO mouse brain for three weeks. Experiment replicated five times. g, Quantification of neuron number and area covered by MAP2 immunoreactivity for co-cultured neurons (four wells per genotype, eight random images taken per well). $\mathbf{h}$, TNF- $\alpha$ level in the co-culture medium measured by ELISA. i, Representative images of primary WT neurons infected with AAV2/8-synapsin-P301S human tau treated with $10 \mu \mathrm{g} \mathrm{ml}^{-1}$ recombinant human ApoE for three weeks. Experiment replicated twice. j, Quantification of neuron number and area covered by MAP2 staining for ApoE-treated neurons (three wells per treatment, eight random images per well). Data expressed as mean \pm s.e.m., one-way ANOVA with Tukey's post hoc test, two-sided. $* P<0.05, * * P<0.01, * * * P<0.001$, **** $P<0.0001$.

(Fig. 4f, g). In addition, a significantly higher level of TNF- $\alpha$ was detected in the neuron/E4 co-culture medium whereas minimal TNF- $\alpha$ levels were detected in the neuron/EKO co-culture medium (Fig. 4h), indicating activated glia probably play an important role in inducing neuronal death. Treatment of P301S tau-expressing neurons with recombinant ApoE, regardless of ApoE isoform, invariably led to reduced neurite arborization and increased neuronal death compared with non-ApoE-treated neurons, and these effects were most prominent with ApoE4 treatment (Fig. 4i, j), indicating that ApoE itself was directly involved in inducing neurotoxicity in P301S tau-expressing susceptible neurons. ApoE has been shown to accumulate in damaged neurons of Pick disease ${ }^{21}$ and in neurofibrillary tangle (NFT)-bearing neurons of Alzheimer disease ${ }^{22}$, suggesting a potential role of ApoE in mediating neurotoxicity under pathological conditions. However, overall neuronal loss or damage after ApoE treatment was much milder than that observed in neuron-glia co-cultures, suggesting an additive role of concomitant neuroinflammation in exacerbating neurodegeneration.

To investigate whether the pro-neurodegenerative effect of ApoE4 also manifests in human primary tauopathies, we assessed post-mortem regional neurodegeneration severity in relation to ApoE genotype in 
individuals with a primary diagnosis of corticobasal degeneration, Pick disease and progressive supranuclear palsy, the three most prevalent sporadic primary tauopathies. Possession of an $\varepsilon 4$ allele was associated with more severe regional neurodegeneration (linear mixed-effects model, estimate $0.31, P=0.035,95 \%$ (CI $0.02,0.59)$ ) after controlling for age, sex, diagnosis, rater, amyloid- $\beta$ deposition, Lewy body disease, tau burden, and TDP-43 inclusion burden (Extended Data Table 1). In addition, we demonstrated greater regional neurodegeneration as a function of tau burden (estimate $0.17, P<0.001$. 95\% CI $(0.14,0.20)$ ) and TDP-43 proteinopathy (estimate $0.22, P=0.003,95 \% \mathrm{CI}(0.07$, $0.37)$ ). By contrast, amyloid- $\beta$ deposition was associated with less severe neurodegeneration (estimate $0.08, P=0.044,95 \%$ CI $(-0.15$, $-0.002)$ ), and Lewy body disease, which was relatively uncommon in this cohort, showed no effect (estimate $-0.01, P=0.87,95 \% \mathrm{CI}(-0.17$, 0.15)) (Extended Data Table 1). We also analysed disease progression in relation to APOE allele status in a cohort of individuals with low CSF amyloid- $\beta 42$ who had symptomatic Alzheimer disease, who invariably possess amyloid- $\beta$ deposition and usually have tau pathology. We found possession of the $\varepsilon 4$ allele was associated with significantly higher rates of clinical disease progression $(P=0.02)$ in a dose-dependent manner. Individuals with one $\varepsilon 4$ allele progressed $14 \%$ faster than non-carriers, and those with two $\varepsilon 4$ alleles progressed $23 \%$ faster (Extended Data Fig. 8). Overall, these findings provide converging evidence in humans that possession of the $\varepsilon 4$ allele worsens neurodegeneration in the setting of a tauopathy, with or without amyloid- $\beta$ pathology.

Taken together, we found ApoE affects neurodegeneration in the context of tau pathology independently of amyloid- $\beta$. ApoE4 resulted in aggravated neurodegeneration whereas the absence of ApoE was strongly neuroprotective. This was probably a direct effect of ApoE modulating tau pathology from an early age, which ultimately led to various tau forms/patterns potentially possessing distinct neurotoxicity. On the other hand, we found ApoE itself, especially ApoE4, was essential to $\mathrm{P} 301 \mathrm{~S}$ neuronal death. With pathological tau accumulation, the presence of ApoE, especially ApoE4, may make neurons more susceptible to degeneration, whereas the absence of ApoE may protect neurons from death. The presence of degenerating neurons appeared to further induce neuroinflammation, which was augmented by ApoE4 owing to its inherently higher innate immune reactivity. While activated microglia may be protective to some extent in the setting of amyloid- $\beta$ pathology, by targeting plaques and reducing dystrophic neurites ${ }^{23,24}$, they could be deleterious in tauopathy by directly targeting injured neurons and by activating toxic A1 astrocytes. Enhanced neuroinflammation associated with ApoE4 may further exacerbate neurodegeneration (Extended Data Fig. 9 for hypothesis summary). These new findings suggest a re-evaluation of the role of ApoE in Alzheimer disease and other tauopathies. Targeting ApoE, especially ApoE4, may be a promising therapeutic approach in reducing tau-mediated neurodegeneration.

Online Content Methods, along with any additional Extended Data display items and Source Data, are available in the online version of the paper; references unique to these sections appear only in the online paper.

\section{Received 25 November 2016; accepted 17 August 2017.}

\section{Published online 20 September 2017.}

1. Holtzman, D. M., Herz, J. \& Bu, G. Apolipoprotein E and apolipoprotein E receptors: normal biology and roles in Alzheimer disease. Cold Spring Harb. Perspect. Med. 2, a006312 (2012)

2. Strittmatter, W. J. et al. Apolipoprotein E: high-avidity binding to beta-amyloid and increased frequency of type 4 allele in late-onset familial Alzheimer disease. Proc. Natl Acad. Sci. USA 90, 1977-1981 (1993).

3. Josephs, K. A. et al. $\beta$-amyloid burden is not associated with rates of brain atrophy. Ann. Neurol. 63, 204-212 (2008)

4. Arriagada, P. V., Growdon, J. H., Hedley-Whyte, E. T. \& Hyman, B. T. Neurofibrillary tangles but not senile plaques parallel duration and severity of Alzheimer's disease. Neurology 42, 631-639 (1992)

5. Williams, D. R. et al. Pathological tau burden and distribution distinguishes progressive supranuclear palsy-Parkinsonism from Richardson's syndrome. Brain 130, 1566-1576 (2007).

6. Strittmatter, W. J. et al. Isoform-specific interactions of apolipoprotein $E$ with microtubule-associated protein tau: implications for Alzheimer disease. Proc. Natl Acad. Sci. USA 91, 11183-11186 (1994).
7. Brecht, W. J. et al. Neuron-specific apolipoprotein e4 proteolysis is associated with increased tau phosphorylation in brains of transgenic mice. J. Neurosci. 24, 2527-2534 (2004)

8. Deming, Y. et al. Genome-wide association study identifies four novel loci associated with Alzheimer's endophenotypes and disease modifiers. Acta Neuropathol. 133, 839-856 (2017)

9. Mishra, A. et al. Gene-based association studies report genetic links for clinical subtypes of frontotemporal dementia. Brain 140, 1437-1446 (2017).

10. Stevens, M. et al. Apolipoprotein E gene and sporadic frontal lobe dementia. Neurology 48, 1526-1529 (1997).

11. Agosta, F. et al. Apolipoprotein $\mathrm{E} \varepsilon 4$ is associated with disease-specific effects on brain atrophy in Alzheimer's disease and frontotemporal dementia. Proc. Natl Acad. Sci. USA 106, 2018-2022 (2009).

12. Engelborghs, S. et al. Dose dependent effect of APOE epsilon 4 on behavioral symptoms in frontal lobe dementia. Neurobiol. Aging 27, 285-292 (2006)

13. Yoshiyama, Y. et al. Synapse loss and microglial activation precede tangles in a P301S tauopathy mouse model. Neuron 53, 337-351 (2007).

14. Simonovitch, S. et al. Impaired autophagy in APOE4 astrocytes. J. Alzheimers Dis. 51, 915-927 (2016).

15. Bales, K. R. et al. Human APOE isoform-dependent effects on brain betaamyloid levels in PDAPP transgenic mice. J. Neurosci. 29, 6771-6779 (2009).

16. Liddelow, S. A. et al. Neurotoxic reactive astrocytes are induced by activated microglia. Nature 541, 481-487 (2017).

17. Liu, X. et al. Inflammasome-activated gasdermin D causes pyroptosis by forming membrane pores. Nature 535, 153-158 (2016)

18. Vitek, M. P., Brown, C. M. \& Colton, C. A. APOE genotype-specific differences in the innate immune response. Neurobiol. Aging 30, 1350-1360 (2009).

19. Gale, S. C. et al. APO $\varepsilon 4$ is associated with enhanced in vivo innate immune responses in human subjects. J. Allergy Clin. Immunol. 134, 127-134 (2014).

20. Zamanian, J. L. et al. Genomic analysis of reactive astrogliosis. J. Neurosci. $\mathbf{3 2}$, 6391-6410 (2012)

21. Farrer, L. A. et al. Allele epsilon 4 of apolipoprotein $E$ shows a dose effect on age at onset of Pick disease. Exp. Neurol. 136, 162-170 (1995).

22. Huang, Y. et al. Apolipoprotein E fragments present in Alzheimer's disease brains induce neurofibrillary tangle-like intracellular inclusions in neurons. Proc. Natl Acad. Sci. USA 98, 8838-8843 (2001).

23. Yuan, P. et al. TREM2 haplodeficiency in mice and humans impairs the microglia barrier function leading to decreased amyloid compaction and severe axonal dystrophy. Neuron 90, 724-739 (2016).

24. Wang, Y. et al. TREM2-mediated early microglial response limits diffusion and toxicity of amyloid plaques. J. Exp. Med. 213, 667-675 (2016).

Supplementary Information is available in the online version of the paper.

Acknowledgements This study was funded by National Institutes of Health (NIH) NS090934 (D.M.H.), P01-AG03991 (D.M.H., J.C.M., A.M.F.), P01-AG026276 (D.M.H., J.C.M., A.M.F.), P50 AG05681 (D.M.H., J.C.M., A.M.F.), the JPB Foundation (D.M.H., B.A.B.), Cure Alzheimer's Fund (D.M.H.), a grant from AstraZeneca (D.M.H., S.M.P.), NIH AG023501 (W.W.S.), AG019724 (W.W.S.), Consortium for Frontotemporal Dementia Research (W.W.S.), Tau Consortium (W.W.S.), NIH K08 AG052648 (S.S.), NIH AG051812 (O.B.), NS088137 (O.B.), National Multiple Sclerosis Society (5092A1) (O.B.), Nancy Davis Foundation Award (O.B.), Amyotrophic Lateral Sclerosis Association (ALSA2087) (O.B.), and NIH K01 NS096719-01 (G.G.). We thank J. Yu for technical assistance in gene expression analysis; N. Barthélemy for assistance in tau phosphorylation analysis; and S. Schindler for assistance in statistical analysis. Data collection and sharing for this project were funded by the Alzheimer's Disease

Neuroimaging Initiative (ADNI) (NIH Grant U01 AG024904) and Department of Defense ADNI (award number W81XWH-12-2-0012). A full list of ADNI funding information is listed in the Supplementary Information.

Author Contributions D.M.H., S.M.P., and Y.S. conceived the study. Y.S., K.Y., and D.M.H. designed the study. Y.S. performed most of the experiments and analysed the data, assisted by K.Y., K.W., G.G., L.Z, M.W.W., J.R., G.R., M.B.F., and H.J.; S.T.S., C.B., and O.B. performed the Nanostring microglial gene expression assay; S.A.L. and B.A.B. performed the astrocytic gene expression assay; W.L. performed the microglia LPS stimulation assay; R.M.T., S.S., L.T.G., B.L.M., W.W.S., J.C.R., and A.L.B. performed data analysis for human primary tauopathies; J.D.A., L.M., C.S., C.X., J.C.M., A.F., and C.C. performed data analysis in human patients with Alzheimer disease; P.M.S. provided the ApoE KI mice. Y.S. and D.M.H. wrote the manuscript. All authors discussed the results and commented on the manuscript. A portion of the human Alzheimer disease data used in preparation of this article was obtained from the ADNI database (http://adni.loni.usc.edu). As such, the investigators within the ADNI contributed to the design and implementation of ADNI and/or provided data but did not participate in analysis or writing of this report. A complete listing of ADNI investigators can be found in the Supplementary Information.

Author Information Reprints and permissions information is available at www.nature.com/reprints. The authors declare competing financial interests: details are available in the online version of the paper. Readers are welcome to comment on the online version of the paper. Publisher's note: Springer Nature remains neutral with regard to jurisdictional claims in published maps and institutional affiliations. Correspondence and requests for materials should be addressed to D.M.H. (holtzman@wustl.edu).

Reviewer Information Nature thanks C. Haass, E. Roberson and the other anonymous reviewer(s) for their contribution to the peer review of this work. 


\section{METHODS}

Animals. Human ApoE2, ApoE3, and ApoE4 KI mice (C57BL/6) were provided by P. M. Sullivan (Duke University) and ApoE KO mice (C57BL/6) were purchased from The Jackson Laboratory (002052). P301S tau transgenic mice (The Jackson Laboratory, 008169) on a B6/C3 background expressing human P301S 1N4R tau driven by PrP promoter were crossed to human ApoE KI mice to generate P301S ${ }^{\mathrm{hE} / \mathrm{hE}}$ mice for all three ApoE isoforms, which were then crossed to ApoE KO mice to generate $\mathrm{P} 301 \mathrm{~S}^{\mathrm{hE} /-}$ mice. Separately, ApoE KI mice were crossed to ApoE $\mathrm{KO}$ mice to generate $\mathrm{hE} /-$ mice. $\mathrm{P} 301 \mathrm{~S}^{\mathrm{hE} /}$ - mice were then crossed with $\mathrm{hE} /-$ mice to generate $\mathrm{P} 301 \mathrm{~S}^{\mathrm{hE} / \mathrm{hE}}, \mathrm{P} 301 \mathrm{~S}^{\mathrm{hE} /-}$, and $\mathrm{P} 301 \mathrm{~S}^{-1-}$ littermates for all three ApoE isoforms. All tau transgenic mice for final analysis were kept at the same generation and had $\sim 90 \%$ C57BL/6 background by congenic test. Only male ApoE homozygote and $\mathrm{KO}$ mice were used for analysis. P301S ApoE KO mice derived from three ApoE isoforms showed no difference in analysis and were combined as one single group (TEKO). WT mice were purchased from Charles River Laboratories (027) on a C57BL/6 background and were bred separately from the P301S/ApoE mice. Owing to a slightly different background of the WT mice, we did not perform statistical analysis comparing WT with other mice. All animal procedures and experiments were performed under guidelines approved by the animal studies committee at Washington University School of Medicine. Sample sizes were chosen on the basis of estimates to provide $80 \%$ power to see significant differences of $20 \%$ or greater. All assessment and analysis of data obtained from mice was performed by someone blind to the genotypes of the animals.

Immunohistochemistry. The left hemi-brain of each mouse was sectioned coronally at $50 \mu \mathrm{m}$ using a freezing sliding microtome. Three sections from each mouse (300 $\mu \mathrm{m}$ apart), corresponding approximately to bregma coordinates $-1.4,-1.7$, and $-2.0 \mathrm{~mm}$ respectively, were used for $\mathrm{p}$-tau staining described in ref. 25 . Briefly, brain sections were washed in Tris-buffered saline (TBS) buffer three times followed by incubation in $0.3 \%$ hydrogen peroxide in TBS for $10 \mathrm{~min}$ at room temperature. After three washes in TBS, sections were blocked with $3 \%$ milk in $0.25 \%$ TBS-X (Triton X-100) for $0.5 \mathrm{~h}$ followed by incubation at $4{ }^{\circ} \mathrm{C}$ overnight with biotinylated AT8 antibody (Thermo Scientific, MN1020B, 1:500). The next day, the slices were developed using a VECTASTAIN Elite ABC HRP Kit (Vector laboratories) following the manufacturer's instructions. Stained sections were imaged by a NanoZoomer digital pathology system and pathology was quantified using Image J. For immunofluorescence, two sections (bregma $-1.7 \mathrm{~mm}$ and $-2.0 \mathrm{~mm}$ for CD68, bregma $-2.3 \mathrm{~mm}$ and $-2.6 \mathrm{~mm}$ for GFAP) from each mouse were used. The sections were washed in TBS three times, and blocked with 3\% BSA in $0.25 \%$ TBS-X for $0.5 \mathrm{~h}$ at room temperature, followed by overnight incubation at $4{ }^{\circ} \mathrm{C}$ with primary antibodies (CD68: AbD SeroTec, MCA1957, 1:500; GFAP: EMD Millipore, MAB3402, 1:2,000). The next day, the sections were washed in TBS and incubated with fluorescence-labelled secondary antibodies (Molecular Probes, 1:500) for $2 \mathrm{~h}$ at room temperature. The slices were then washed and mounted in ProLong Gold Antifade mounting medium (Molecular Probes, P36931). Images were taken with an epi-fluorescence microscope at $4 \times$ magnification and quantified using MetaMorph.

Volumetric analysis. Every sixth coronal brain section ( $300 \mu \mathrm{m}$ between sections) starting rostrally at bregma $+2.1 \mathrm{~mm}$ to the dorsal end of the hippocampus at bregma $-3.9 \mathrm{~mm}$ were mounted for each mouse. All mounted sections were stained with $0.1 \%$ Sudan black in $70 \%$ ethanol at room temperature for $20 \mathrm{~min}$, then washed in $70 \%$ ethanol for $1 \mathrm{~min}$, three times. The sections were finally washed in Milli-Q water three times and coverslipped with Fluoromount. The stained slices were imaged with the NanoZoomer and areas of interest were traced and measured in each slice using the NDP viewer. The volume was calculated using the following formula: volume $=($ sum of area $) \times 0.3 \mathrm{~mm}$. For hippocampus and posterior lateral ventricle, quantification started from bregma $-1.1 \mathrm{~mm}$ and ended at bregma $-3.9 \mathrm{~mm}$. For piriform/entorhinal cortex, quantification started at bregma $-2.3 \mathrm{~mm}$ and ended at bregma $-3.9 \mathrm{~mm}$. All staining and analysis of data obtained were performed by someone blind to the genotypes of the animals being assessed. Neuronal layer thickness measurement. Three sections (bregma $-1.4,-1.7$, and $-2.0 \mathrm{~mm}$ ) from each mouse were mounted and stained in cresyl violet for $5 \mathrm{~min}$ at room temperature. The slices were then sequentially dehydrated in $50 \%, 70 \%$, $95 \%$ (three times) and $100 \%$ ethanol (twice) for $1 \mathrm{~min}$, then cleared in xylene for $4 \mathrm{~min}$ (twice), and coverslipped in cytoseal60 (Thermo Fisher Scientific, 8310-16). The thicknesses of the CA1 pyramidal cell layer and dentate gyrus granular cell layer were measured by drawing a scale perpendicular to the cell layer at two spots in all three slices and taking the average value for each mouse. All staining and analysis of data obtained were performed by someone blind to the genotypes of the animals being assessed.

Brain extraction. Mouse cortex was processed in RAB, RIPA, and 70\% formic acid buffer sequentially as described previously ${ }^{25}$ with modifications. Briefly, the tissue was weighed and homogenized using a pestle with $10 \mu \mathrm{l}$ buffer per $1 \mathrm{mg}$ tissue in RAB buffer (100 mM MES, $1 \mathrm{mM}$ EGTA, $0.5 \mathrm{mM} \mathrm{MgSO}_{4}, 750 \mathrm{mM} \mathrm{NaCl}, 20 \mathrm{mM}$
$\mathrm{NaF}, 1 \mathrm{mM} \mathrm{Na} \mathrm{VO}_{4}, \mathrm{pH}=7.0$, supplemented by protease inhibitors (Complete, Roche) and phosphatase inhibitors (PhosSTOP, Roche)). After centrifugation at $50,000 \mathrm{~g}$ for $20 \mathrm{~min}$, the supernatant was taken as the RAB-soluble fraction and the pellet was dissolved in RIPA buffer $(150 \mathrm{mM} \mathrm{NaCl}, 50 \mathrm{mM}$ Tris, $0.5 \%$ deoxycholic acid, $1 \%$ Triton X-100, 0.1\% SDS, $5 \mathrm{mM}$ EDTA, $20 \mathrm{mM} \mathrm{NaF}, 1 \mathrm{mM} \mathrm{Na}_{3} \mathrm{VO}_{4}$, $\mathrm{pH}$ 8.0, supplemented by Complete and PhosSTOP) at $10 \mu \mathrm{l}$ buffer per $1 \mathrm{mg}$ tissue by sonication. After centrifugation at $50,000 \mathrm{~g}$ for $20 \mathrm{~min}$, the supernatant was taken as the RIPA-soluble fraction. The pellet was sonicated in $70 \%$ formic acid at $10 \mu \mathrm{l}$ buffer per $1 \mathrm{mg}$ tissue, and centrifuged at $50,000 \mathrm{~g}$ for $20 \mathrm{~min}$. The supernatant was taken as the formic-acid-soluble fraction. All fractions were stored in $-80^{\circ} \mathrm{C}$ until analysed.

ELISA. Human tau and ApoE ELISAs were performed as described previously ${ }^{25}$ For coating antibodies, Tau5 (gift from L. Binder, Northwestern University, Chicago, Illinois, USA) was used for human tau ELISA, and HJ15.6 (in-housemade anti-human ApoE antibody) was used for human ApoE ELISA. For detection antibodies, biotinylated HT7 (Thermo Fisher Scientific, MN1000B) was used for tau ELISA and biotinylated HJ15.4 (in house-made anti-human ApoE antibody) was used for human ApoE ELISA. All measurements and analysis of data obtained were performed by someone blind to the genotypes of the animals being assessed. Western blot. Mouse cortex was homogenized by sonication in RIPA buffer supplemented with Complete and PhosSTOP. Samples were separated by $4-12 \%$ NuPAGE (Invitrogen) gel using MOPS buffer and transferred to nitrocellulose membranes. Primary antibodies anti-GFAP (Millipore, MAB 3402), anti-GAPDH (Abcam, ab9484), anti-ApoE (Abcam, ab24139), anti- $\alpha$ tubulin (Sigma, T5168), and HRP-conjugated secondary antibodies (Santa Cruz) were used for detection. Membranes were developed using Lumigen TMA6 (GE Healthcare).

qRT-PCR for human tau. RNAs were isolated from mouse cortex using RNeasy Mini Kit (QIAGEN,74104), and reverse transcribed to complementary DNA using a high-capacity RNA-to-cDNA kit (Applied Biosystems, 4387406). Quantitative PCR was performed using Taqman primers and probes with Taqman Universal PCR Master Mix (Applied Biosystems, 4304437). Quantitative PCR and analysis of data obtained were performed by someone blind to the genotypes of the animals being assessed.

Nanostring gene expression assay. RNAs were isolated from mouse hippocampus using an RNeasy Mini Kit (QIAGEN, 74104). We performed nCounter multiplexed target profiling of 534 microglial transcripts with a custom-designed microglial gene chip (MG550) using the Nanostring nCounter technology (http://nanostring. com), which allows analysis of up to 800 genes from a single sample. Selection of genes was based on genes and proteins specifically or highly expressed in adult homeostatic microglia ${ }^{26}$ plus 150 inflammation-related genes that were significantly affected in EAE, APP-PS1 and SOD1 mice ${ }^{27}$. Using this signature, we generated a new version (v3) of Nanostring-based microglia chip termed MG550. For each sample, 100 ng RNA was used for hybridization, and all data were normalized to six housekeeping genes: Cltc, Gapdh, Gusb, Hprt1, Pgk1, and Tubb5. All gene expression assays and analysis of data obtained were performed by someone blind to the genotypes of the animals being assessed.

Microfluidic qRT-PCR for astrocytic genes. Total RNA was extracted from mouse cortex using an RNeasy Plus kit (Qiagen) and microfluidic qRT-PCR was performed as described ${ }^{16}$

Primary neuron culture and recombinant ApoE treatment. Primary neurons were obtained from E17 WT mouse (Charles River, 022) fetuses. Hippocampi were dissected in calcium- and magnesium-free Hanks' Balanced Salt solution (HBSS) with careful strip of meninges. Tissue was digested in HBSS containing $0.25 \%$ trypsin (GIBCO 15090-046) and $0.2 \mathrm{mg} \mathrm{ml}^{-1}$ DNase (Sigma DN-25) at $37^{\circ} \mathrm{C}$ for $10 \mathrm{~min}$, and was dissociated in HBSS containing $0.4 \mathrm{mg} \mathrm{ml}^{-1}$ DNase using a fire-polished Pasteur glass pipette and filtered through a $70-\mu \mathrm{m}$ nylon mesh. Filtered material was pelleted at $1,000 \mathrm{~g}$ for $5 \mathrm{~min}$, washed with neurobasal medium (neurobasal $+1 \times \mathrm{B} 27+1 \times$ penicillin/streptomycin $+1 \times$ L-glutamine) once, and infected with AAV2/8-synapsin-P301S tau virus for $3 \mathrm{~h}$ on ice. Cells were then pelleted, washed, and plated onto 24 -well tissue culture plates over glass cover slips that had been coated with $10 \mu \mathrm{g} \mathrm{ml}^{-1}$ poly-L-lysine (PLL, Sigma, P2636) at a density of 20,000 cells per well in neurobasal medium. For all cell culture experiments, only the central wells of the plates were used with the peripheral wells filled with $1 \mathrm{ml}$ autoclaved double-distilled $\mathrm{H}_{2} \mathrm{O}$ to avoid different rates of medium evaporation during the culture. Recombinant ApoE2, ApoE3, and ApoE4 (Leinco, $\mathrm{A} 215, \mathrm{~A} 218, \mathrm{~A} 219)$ were added to the medium $\left(10 \mu \mathrm{g} \mathrm{ml}^{-1}\right)$ at the time of plating. Neurons were kept for 3 weeks with addition of $200 \mu \mathrm{l}$ fresh recombinant ApoEcontaining medium $\left(10 \mu \mathrm{g} \mathrm{ml}^{-1}\right)$ each well every week, and were then stained with MAP2 antibody (Thermo Fisher Scientific, OSM00030W) for analysis and with HT7B (Thermo Fisher Scientific, MN1000B) antibody to confirm tau expression. Glia-neuron co-culture. E2, E3, E4, and EKO glia were obtained from P2 pups from the respective human ApoE KI or ApoE KO mice. Cortex was dissected and dissociated in the same way as with neuron isolation. Cells were plated in glial 
medium (DMEM $+10 \%$ FBS $+1 \times$ Pen/strep $+1 \times$ Glutamax). Once monolayers were confluent, cells were replated in 24-well plates over glass cover slips coated with geltrex (Gibco, A1413201) at a density of 75,000 cells per well, and allowed to grow for 2 days in glia medium. The glia were then washed with $1 \mathrm{ml}$ neurobasal medium (Neurobasal $+2 \%$ B27 $+1 \times$ penicillin/streptomycin $+1 \times$ L-glutamine) and placed in $400 \mu \mathrm{l}$ neurobasal medium before use. Primary neurons expressing P301S tau were prepared as described above, and were directly plated on top of the glia at a density of 20,000 cells per well. The co-cultures were kept for 3 weeks with addition of $200 \mu \mathrm{l}$ fresh neurobasal medium each well every week. Neurons were then stained with MAP2 antibody (Thermo Fisher Scientific, OSM00030W) for analysis and with HT7B (Thermo Fisher Scientific, MN1000B) antibody to confirm tau expression.

Primary microglia culture, LPS stimulation and cytokine measurement. Mixed glia culture from ApoE2, ApoE3, and ApoE4 KI mice were prepared as described above in DMEM/F12 medium supplemented with $10 \%$ FBS and $5 \mathrm{ng} \mathrm{ml}^{-1}$ granulocyte-macrophage colony-stimulating factor. Loosely attached microglia were harvested at DIV12 by shaking for $2 \mathrm{~h}$ at 400 r.p.m. and then seeded onto 12 -well tissue culture plates coated with PLL at a density of $1 \times 10^{6}$ cells per well. The next day, seeded microglia were washed in serum-free medium (SFM-DMEM/ $\mathrm{F} 12+0.2 \% \mathrm{BSA}+1 \times$ penicillin/streptomycin) and placed in $450 \mu \mathrm{l}$ serum-free medium. Cells were then treated with $1 \mathrm{ng} \mathrm{ml}^{-1}$ LPS (Sigma, L5293) for $24 \mathrm{~h}$. Media were collected and spun down at 10,000 g for $10 \mathrm{~min}$. Supernatants were collected and assayed for mouse TNF- $\alpha$, IL- $1 \alpha$, and IL- $1 \beta$ using single cytokine ELISAs (Bon Opus Biosciences) according to the manufacturer's instructions. All cytokine levels were normalized to microglial protein levels determined by BCA assay.

Immunocytochemistry. Cells were fixed in DPBS containing 4\% PFA and 4\% sucrose at room temperature for $10 \mathrm{~min}$ and permeabilized with $0.3 \%$ PBST for $10 \mathrm{~min}$. After blocking in $0.1 \%$ PBS-Triton X-100 containing 3\% BSA and $3 \%$ goat serum for $30 \mathrm{~min}$ at room temperature, cells were incubated in primary antibodies overnight at $4{ }^{\circ} \mathrm{C}$. The next day, cells were washed with $0.1 \%$ PBST three times and incubated in secondary antibodies for $1 \mathrm{~h}$ at room temperature. Cells were then washed three times in $0.1 \%$ PBST and mounted in ProLong Gold Antifade mounting medium (Molecular probes, P36931). Images were taken with an epi-fluorescence microscope at $10 \times$ magnification and quantified using MetaMorph. Staining and analysis of immunocytochemistry data obtained were performed by someone blind to the genotypes of the animals being assessed.

Study of neurodegeneration in human primary tauopathies. We searched the University of California, San Francisco (UCSF) Neurodegenerative Disease Brain Bank database for patients with $A P O E$ genotyping and a pathological diagnosis of corticobasal degeneration, Pick disease, or progressive supranuclear palsy, the three most common sporadic forms of frontotemporal lobar degeneration-tau. Authorization for autopsy was provided by patients' next-of-kin, and procedures were approved by the UCSF Committee on Human Research. We constrained our search to patients autopsied since 2007, when systematic, prospective recording of neuropathological findings began. Such data were available from 29 patients with corticobasal degeneration, 17 with Pick disease, and 33 with progressive supranuclear palsy. Demographics and relevant variables are shown in Extended Data Table 1a.

Post-mortem brains were processed as previously described ${ }^{28}$. Fixed tissue slabs were dissected into blocks representing dementia-relevant brain regions, embedded in paraffin wax, and cut into $8 \mu \mathrm{m}$-thick sections. Regional neurodegeneration was assessed using haematoxylin and eosin-stained sections. Neuronal loss, astrogliosis, and microvacuolation were semi-quantitatively graded as absent $(0)$, mild (1), moderate (2), or severe (3). A similar scheme was used to rate amyloid- $\beta$ deposits (neuritic plaques, diffuse plaques, and vascular amyloid-beta deposits) ${ }^{29}$, tau inclusions (neurofibrillary tangles, Pick bodies, globose tangles, neuronal cytoplasmic inclusions (other than those listed previously), tufted astrocytes, thorny astrocytes, astrocytic plaques, neuropil threads, and other glial cytoplasmic inclusions), $\alpha$-synuclein inclusions (Lewy bodies and Lewy neurites), and TDP-43 inclusions (neuronal intranuclear inclusions, neuronal cytoplasmic inclusions, dystrophic neurites, glial cytoplasmic inclusions). Semi-quantitative grading was performed by someone blind to APOE genotype. Composite scores of neurodegeneration (ND score) $[($ microvacuolation + astrogliosis $) / 2)+$ neuronal loss $]$ and for tau, amyloid- $\beta, \alpha$-synuclein, and TDP- 43 were generated for each region by summing scores across inclusion types for each pathological protein.

We limited analyses to regions that had been stained for all four pathological proteins. This approach yielded a total of 609 regional assessments across the 79 patients, with an average of 8 regions per patient. Hippocampal CA1-4, subiculum, dentate gyrus, and entorhinal cortex were the most commonly assessed regions and available in 72 out of 79 patients. Additional regions available from no fewer than 15 patients included midbrain subregions (oculomotor nucleus, tectum, periaqueductal grey, dorsal raphe, and substantia nigra), anterior cingulate cortex, and middle frontal gyrus (Extended Data Table 1b). A linear mixed-effects model conducted at the brain regional level was used to determine the influence of multiple predictors on composite neurodegeneration scores. APOE $\varepsilon 4$ carrier status was used as the binary predictor of interest; additional fixed-effects in the model included tau, amyloid- $\beta$, TDP- 43 , and $\alpha$-synuclein composite scores; sex; age; pathological diagnosis; rater; and brain region. Patient identity was modelled as a random effect. The primary hypothesis was that possession of an ApoE $\varepsilon 4$ allele would be associated with greater regional neurodegeneration after controlling for tau pathological burden and other relevant predictors.

Analysis of clinical disease progression in human Alzheimer disease patients. Participants in this study were part of two different longitudinal studies, the Knight Alzheimer Disease Research Center (ADRC) at Washington University and the ADNI. Details of the Knight ADRC and ADNI studies are listed in the Supplementary Information. All procedures were approved by the Washington University Human Research Protection Office, and written informed consent was obtained from each participant.

Statistical analysis. The experiments were not randomized. Unless explicitly stated, all mouse and cell culture data are shown as mean \pm s.e.m. Differences between groups were evaluated by one-way ANOVA with post hoc Tukey's multiple comparisons tests. For all experiments, data normality was analysed using a D'Agostino-Pearson omnibus normality test. For data not following normal distribution, a Kruskal-Wallis test with Dunn's multiple comparison tes was performed for statistical analysis, and is stated in the figure legends. GraphPad Prism version 6.00 for Windows (GraphPad Software) was used for these analyses and to create the plots. For statistical analysis of the p-tau staining pattern distribution, two-sided Fisher's exact tests were performed using the 'fisher.test' function in R version 3.4.0 (The R Foundation for Statistical Computing) for all four TE groups together, as well as for individual comparisons between each two groups. Data availability. Source data of graphs plotted in Figs 1-4 and Extended Data Figs 1-6 are available as source data files. Other data are available from the corresponding author upon reasonable request. Nanostring gene list, gene pathway analysis, and fully scanned western blot gels can be found in the Supplementary Information.

25. Yanamandra, K. et al. Anti-tau antibodies that block tau aggregate seeding in vitro markedly decrease pathology and improve cognition in vivo. Neuron $\mathbf{8 0}$ 402-414 (2013).

26. Butovsky, O. et al. Identification of a unique TGF- $\beta$-dependent molecular and functional signature in microglia. Nat. Neurosci. 17, 131-143 (2014).

27. Butovsky, O. et al. Targeting miR-155 restores abnormal microglia and attenuates disease in SOD1 mice. Ann. Neurol. 77, 75-99 (2015).

28. Grinberg, L. T. et al. Argyrophilic grain disease differs from other tauopathies by lacking tau acetylation. Acta Neuropathol. 125, 581-593 (2013).

29. Hyman, B. T. et al. National Institute on Aging-Alzheimer's Association guidelines for the neuropathologic assessment of Alzheimer's disease. Alzheimers Dement. 8, 1-13 (2012). 


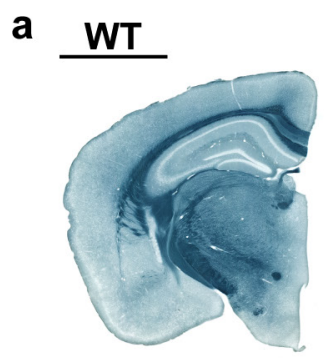

b
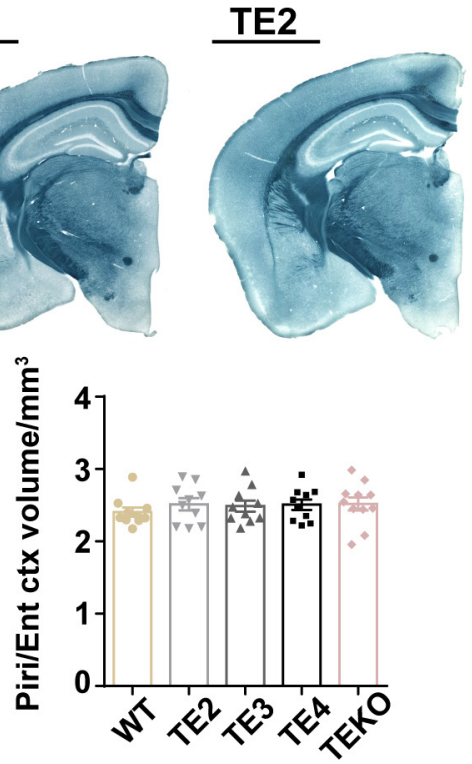

TE3

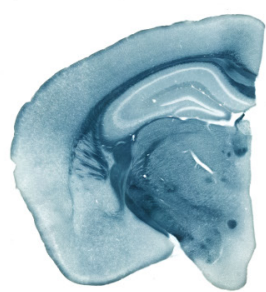

है

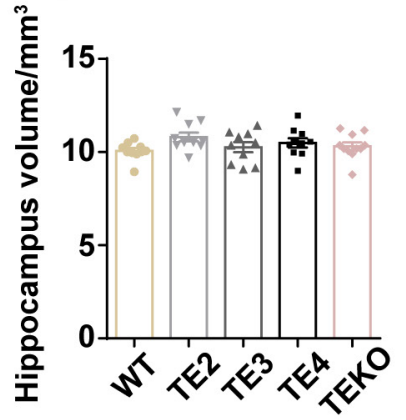

TE4

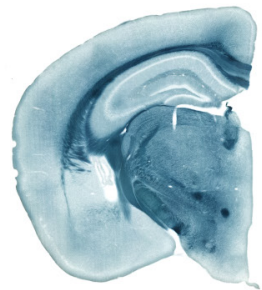

TEKO

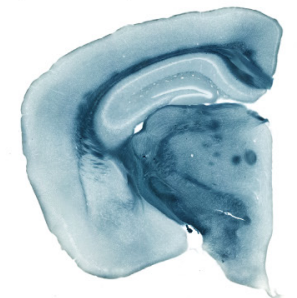

C WT

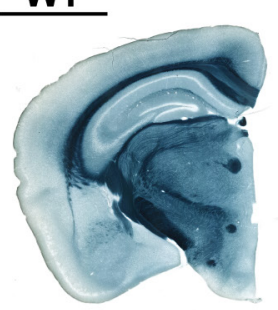

d
E2

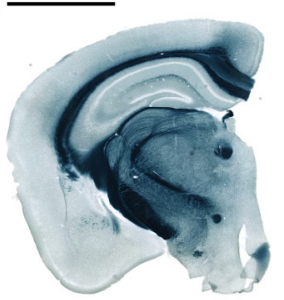

E3
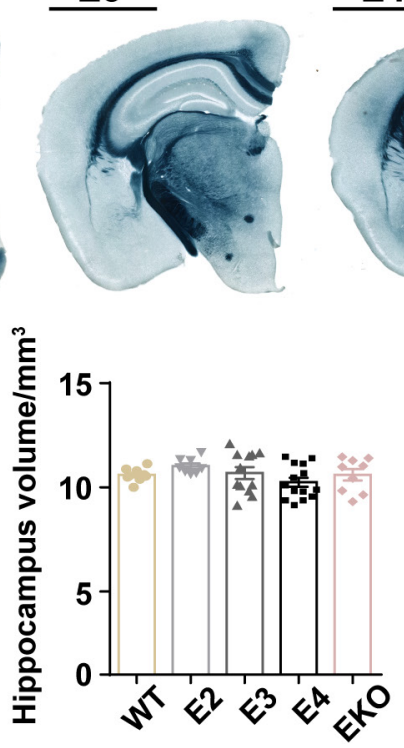

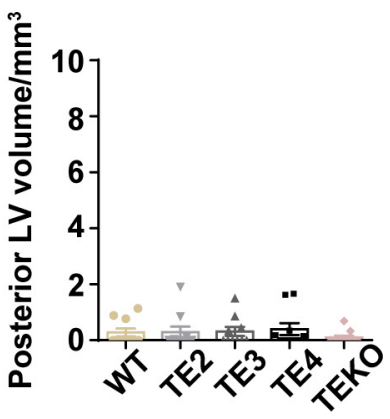

E4

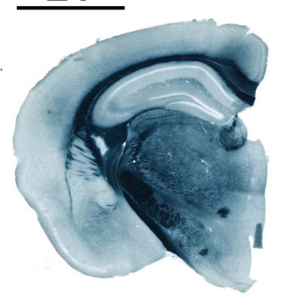

EKO

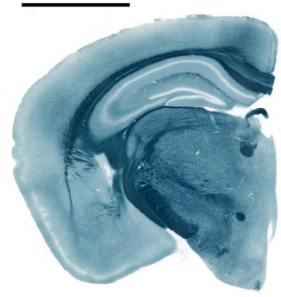

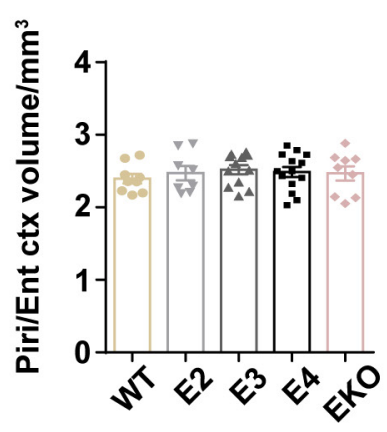

Extended Data Figure 1 | No brain atrophy or brain volume differences in 3-month-old TE mice or 9-month-old non-tau transgenic mice.

a, Representative images of 3-month-old TE mouse brains (WT, $n=10$; TE2, $n=10$; TE3, $n=10$; TE4, $n=10$; TEKO, $n=11$ ) $\mathbf{b}$, Quantification of the piriform/entorhinal cortex, hippocampus, and posterior lateral ventricle volume in 3-month-old TE mice. c, Representative images of 9-month-old non-tau transgenic mouse brains (WT, $n=9$; E2, $n=8$;
E3, $n=12$; E4, $n=14$; EKO, $n=9$ ). d, Quantification of the piriform/ entorhinal cortex, hippocampus, posterior lateral ventricle volume in 9-month-old non-tau transgenic mice. Data expressed as mean \pm s.e.m.; one-way ANOVA with Tukey's post hoc test (two-sided) was used for statistical analysis. Kruskal-Wallis test with Dunn's multiple comparisons test was performed for analysis of posterior lateral ventricle volume. 
a
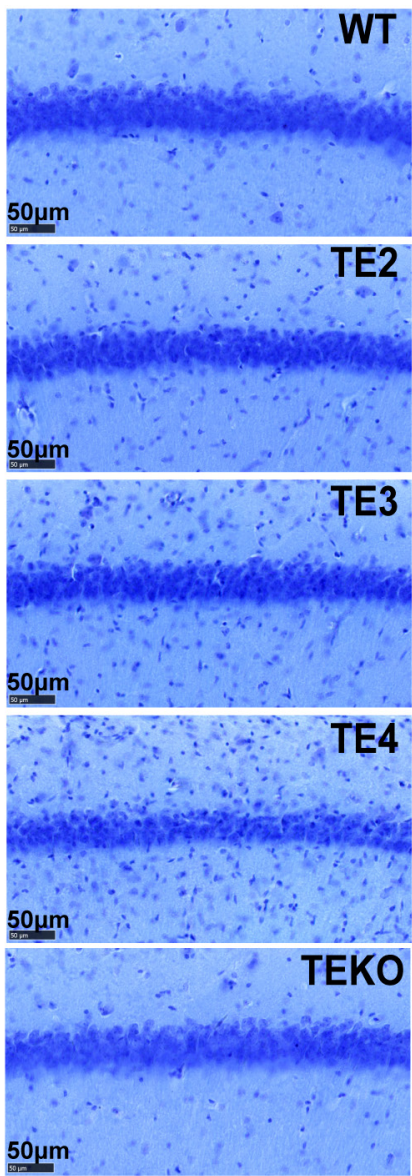

Extended Data Figure $2 \mid$ ApoE4 leads to more severe neuronal loss in the CA1 region of hippocampus in 9-month-old P301S mice.

a, Representative images of 9-month-old TE mouse brain stained with cresyl violet. $\mathbf{b}$, Thickness of the CA1 pyramidal neuronal layer (WT, $n=7$; TE2, $n=14$; TE3, $n=11$; TE4, $n=17$; TEKO, $n=16$ ). Data expressed as b

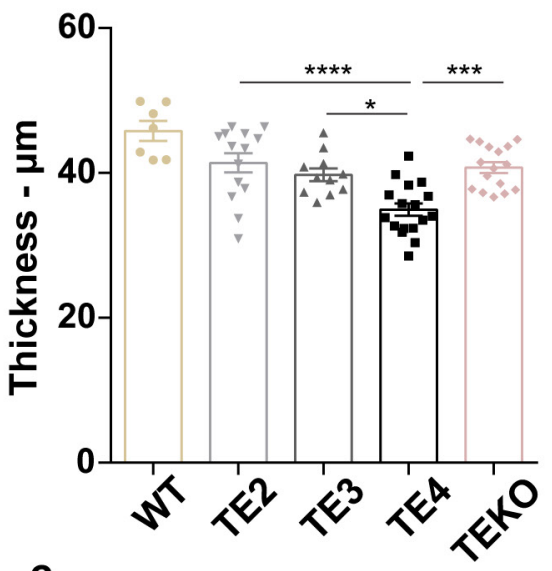

C

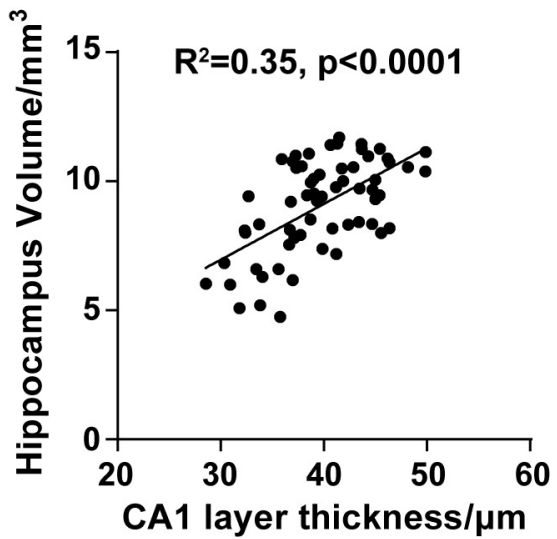

mean \pm s.e.m.; one-way ANOVA with Tukey's post hoc test (two-sided). c, Correlation between CA1 neuronal layer thickness and hippocampal volume. $N=62$ biologically independent animals. Pearson correlation analysis (two-sided), $P<0.0001, R^{2}=0.35 . * P<0.05, * * P<0.01$, $* * * P<0.001, * * * * P<0.0001$. 
a
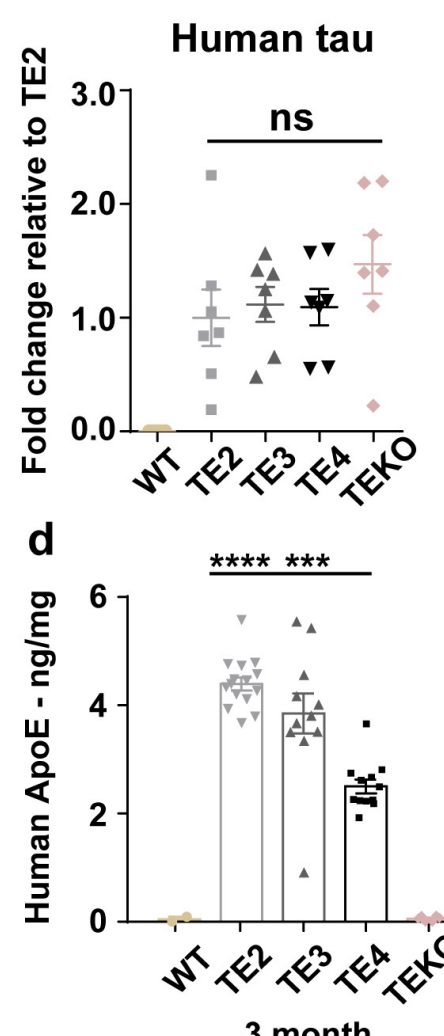

3 month

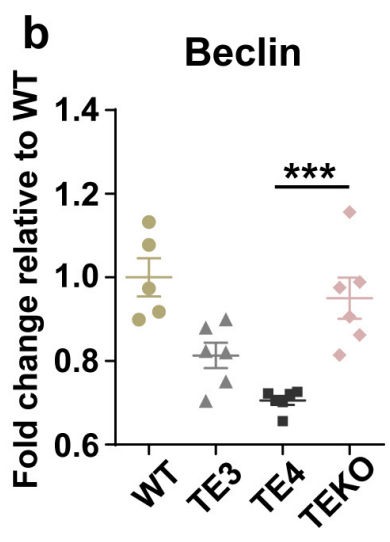

$\star \star * \star * * * * *$
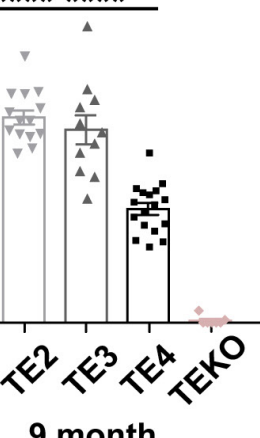

Extended Data Figure 3 | Elevated tau level in TE4 mice is not due to tau synthesis differences, and is probably caused by impairment of autophagy-mediated tau clearance. a, qPCR result for human tau in 9-month-old TE mouse cortex (WT, $n=5$; TE2, TE3, TE4, TEKO, $n=7)$. b, c, Nanostring analysis for autophagy-related gene expression in (b) 9-month-old TE mouse hippocampus and (c) 9-month-old nontau transgenic ApoEKI or ApoEKO mouse hippocampus ( $n=5$ or 6 per group). d, Human ApoE levels in the RAB fraction of 3-month-old

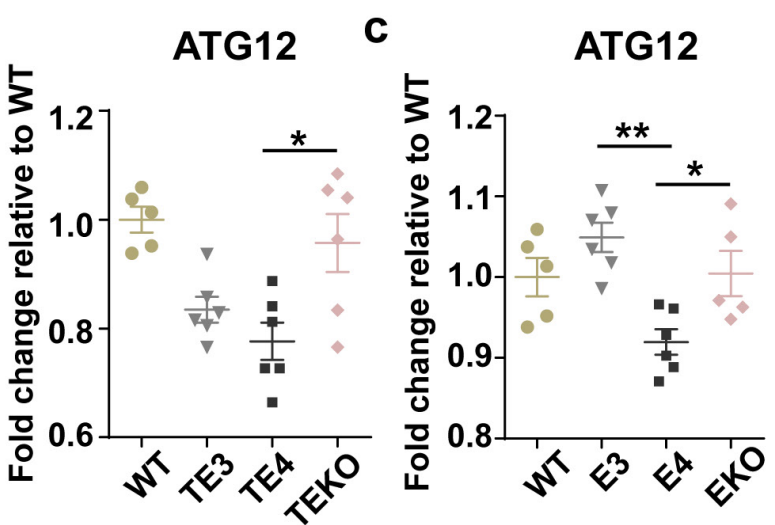

e

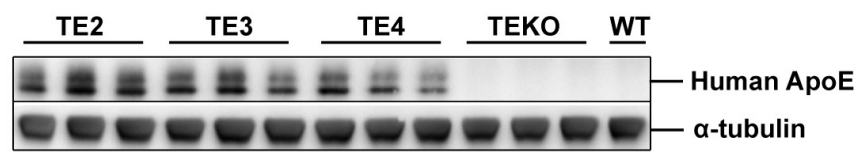

(WT, $n=2$; TE2, $n=15$; TE3, $n=11$; TE4, $n=12$; TEKO, $n=6$ ) and 9-month-old (WT, $n=5$; TE2, $n=14$; TE3, $n=11$; TE $4, n=17$; TEKO, $n=7$ ) TE mouse brain lysates were measured by ELISA. e, Nine-monthold TE mouse cortex was lysed in RIPA buffer without fractionation and total ApoE level was assessed by western blot $(n=3)$. For gel source data, see Supplementary Fig. 2. Data expressed as mean \pm s.e.m.; one-way ANOVA with Tukey's post hoc test (two-sided) was used for statistical analysis. $* P<0.05, * * P<0.01, * * * P<0.001, * * * * P<0.0001$. 
a

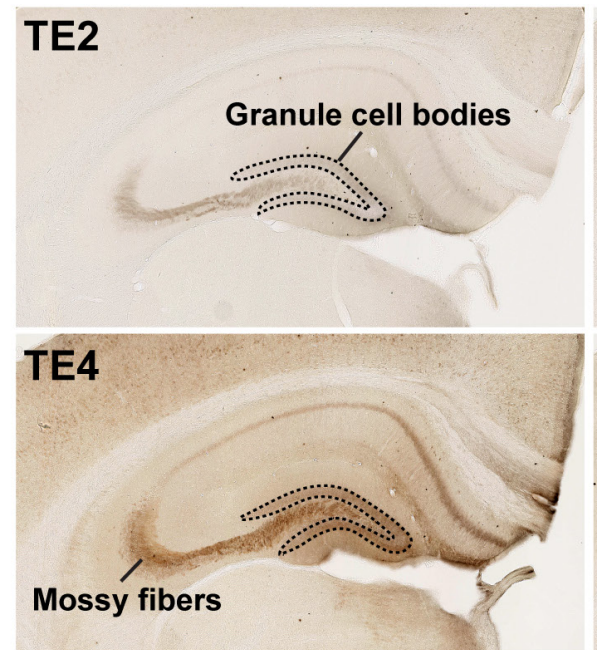

\section{TE3}

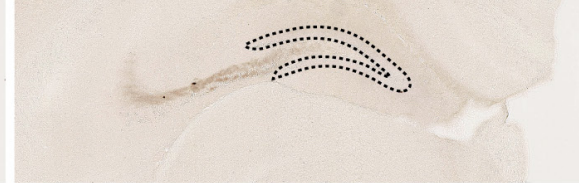

\section{TEKO}

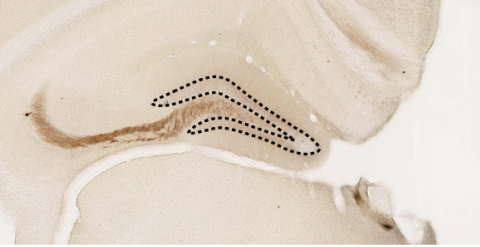

b

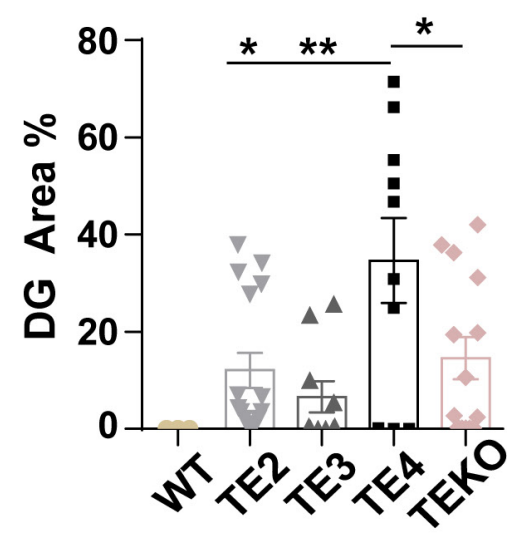

Extended Data Figure 4 | ApoE4 promotes pathological tau redistribution from axons to cell bodies at an early age. a, AT8 staining for 3-month-old TE mouse hippocampus. Dotted outline surrounds the dentate gyrus (DG) granule cell bodies. b, Quantification of AT8 covered area in the dentate gyrus cell body region (TE2, $n=16$; TE3, $n=10$; TE4, $n=10$; TEKO, $n=14$ ). Data expressed as mean \pm s.e.m.; one-way ANOVA with Tukey's post hoc test (two-sided). $* P<0.05, * * P<0.01$, $* * * P<0.001, * * * * P<0.0001$. 


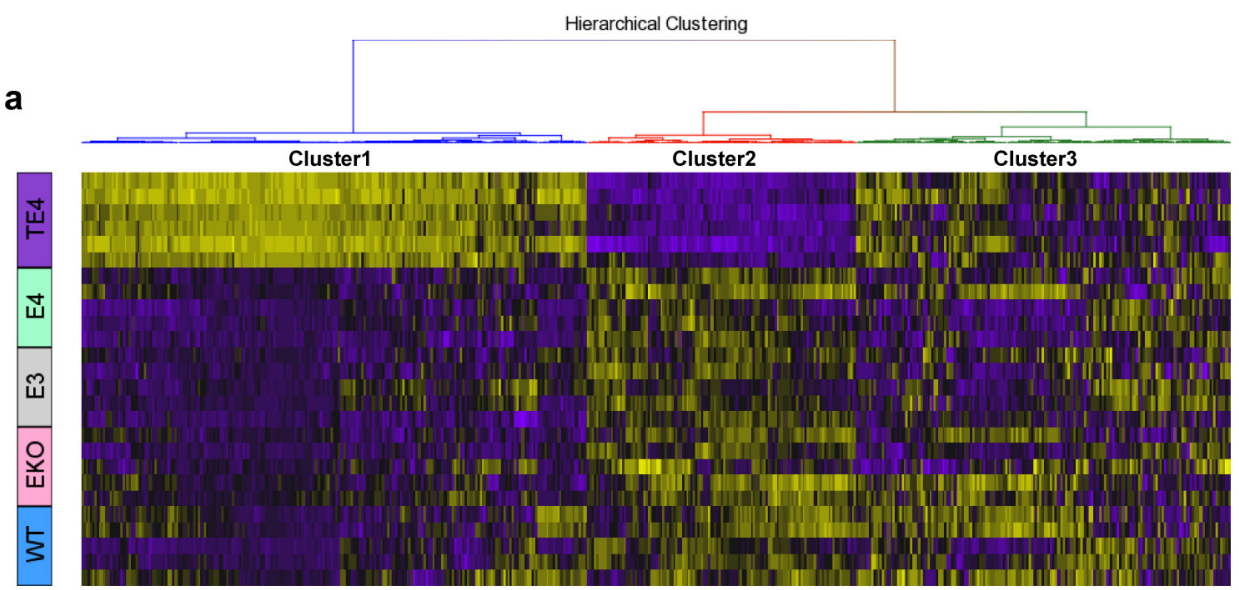

$-3.59$

0.00 b

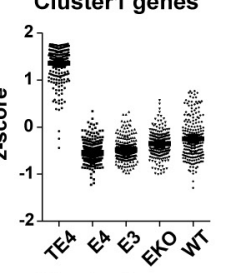

Cluster2 genes

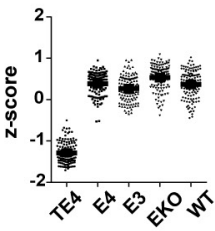

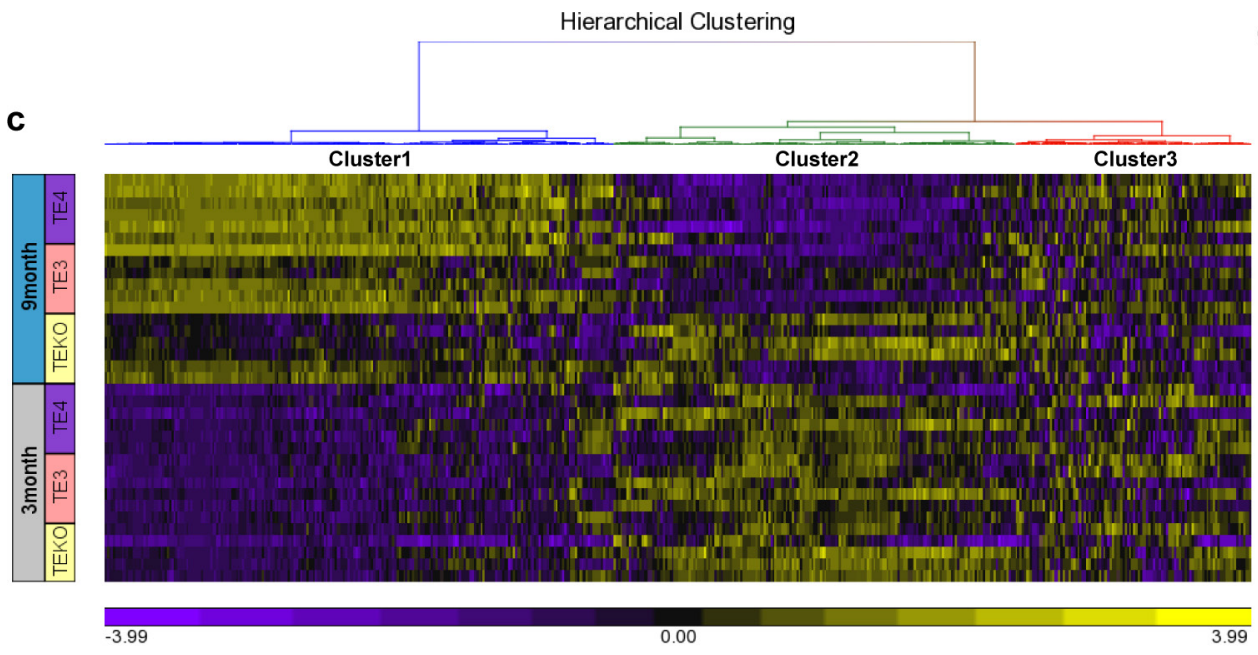

d
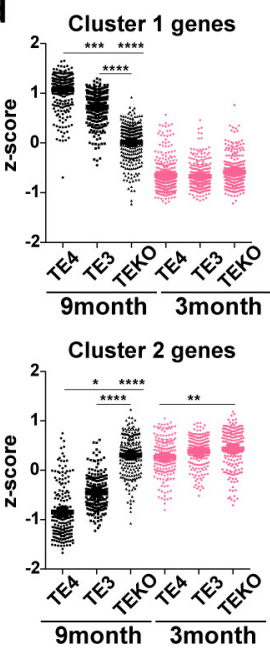

Extended Data Figure 5 | No or minimal change of microglial gene expression in 3-month-old TE mice or 9-month-old non-tau transgenic mice despite significant changes in 9-month-old TE mice. a, Nanostring analysis for microglial gene expression in 9-month-old TE4 mice and 9 -month-old non-tau transgenic mice $(n=5$ or 6$)$. Heatmap generated by hierarchical gene clustering on the basis of genotypes (horizontal: 534 microglial genes; vertical: individual mouse samples) b, Z-score of genes

from cluster 1 or cluster 2 categories. c, Nanostring analysis for microglial gene expression in 9-month-old and 3-month-old TE mice ( $n=5$ or 6$)$. d, $Z$-score of genes from cluster 1 or cluster 2 categories. Kruskal-Wallis test with Dunn's multiple comparisons test was performed for statistical analysis. Data expressed as mean \pm s.e.m. $* P<0.05, * * P<0.01$, $* * * P<0.001, * * * * P<0.0001$. 

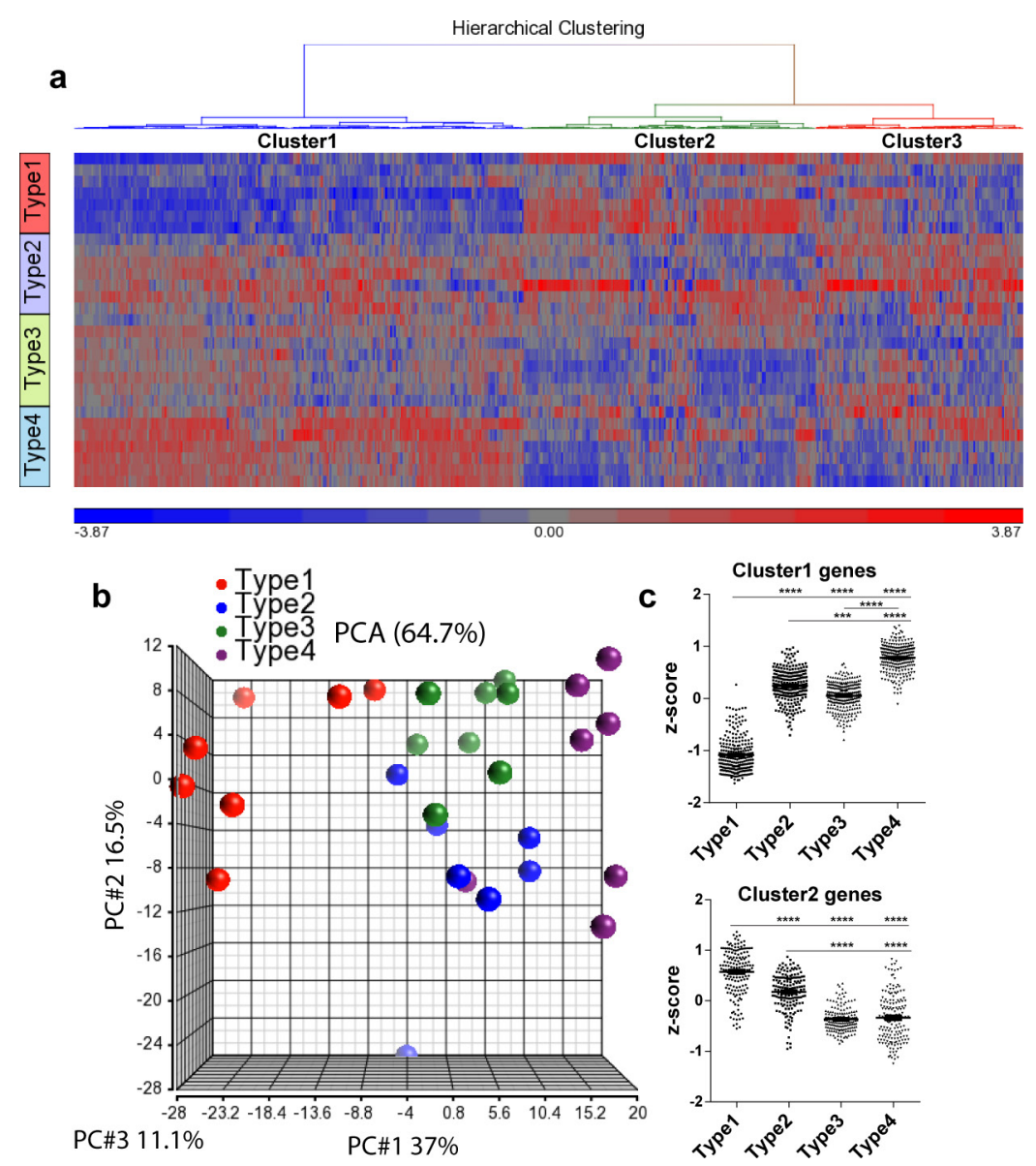

Extended Data Figure 6 | P-tau staining patterns are associated with distinct microglial activation profiles. a, Heatmap generated by hierarchical gene clustering on the basis of $\mathrm{p}$-tau staining types for 9-month-old TE mice ( $n=7$ or 8 ). b. Principle component analysis (PCA) of microglial gene expression profile for $\mathrm{p}$-tau staining types. $\mathrm{c}, Z$-score of genes from cluster 1 or cluster 2 categories. Kruskal-Wallis test with Dunn's multiple comparisons test was performed for statistical analysis. Data expressed as mean \pm s.e.m. $* P<0.05$, $* * P<0.01$, $* * * P<0.001$, $* * * * P<0.0001$. 


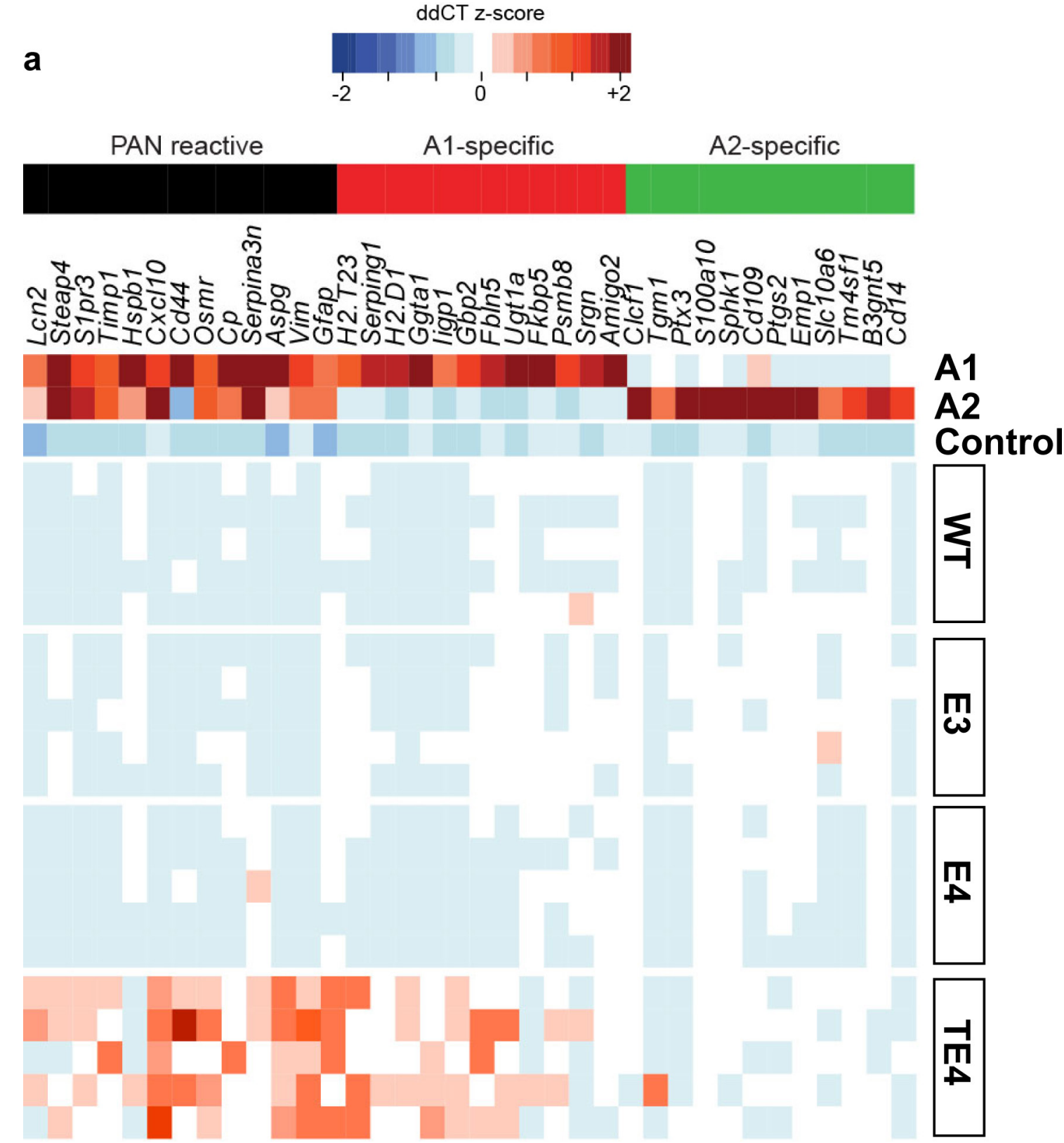

Extended Data Figure $7 \mid$ No activation of A1 astrocytic genes in 9-month-old non-tau transgenic mice. a, Microfluidic qRT-PCR for activated astrocytic genes in 9-month-old TE4 mice and 9-month-old non-tau transgenic WT and human ApoE KI mice $(n=5)$. A1-specific: genes activated only by LPS; A2-specific: genes activated only by ischaemia; PAN reactive: genes activated by either LPS or ischaemia. 


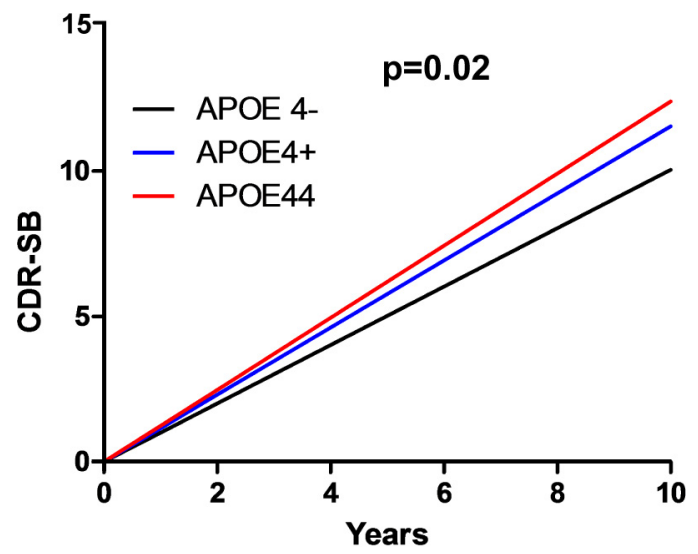

Progression rate:

ApoE4+ > ApoE4- by $14 \%$

ApoE44 > ApoE4- by $23 \%$

Extended Data Figure 8 | Possession of $\varepsilon 4$ allele accelerates the rate of disease progression in patients with Alzheimer disease. Disease progression rate in a cohort of 592 CSF biomarker confirmed individuals with symptomatic Alzheimer disease from two different longitudinal studies, the Knight Alzheimer's Disease Research Center (ADRC) at Washington University and the ADNI. Data generated on the basis of the clinical dementia rating sum of boxes (CDR-SB) scores. Possession of the $\varepsilon 4$ allele significantly accelerated disease progression $(P=0.02)$, with one $\varepsilon 4$ allele increasing progression rate by $14 \%$ and two $\varepsilon 4$ alleles increasing the rate by $23 \%$ compared with non-carriers (lineal mixed model, two-sided). 
a

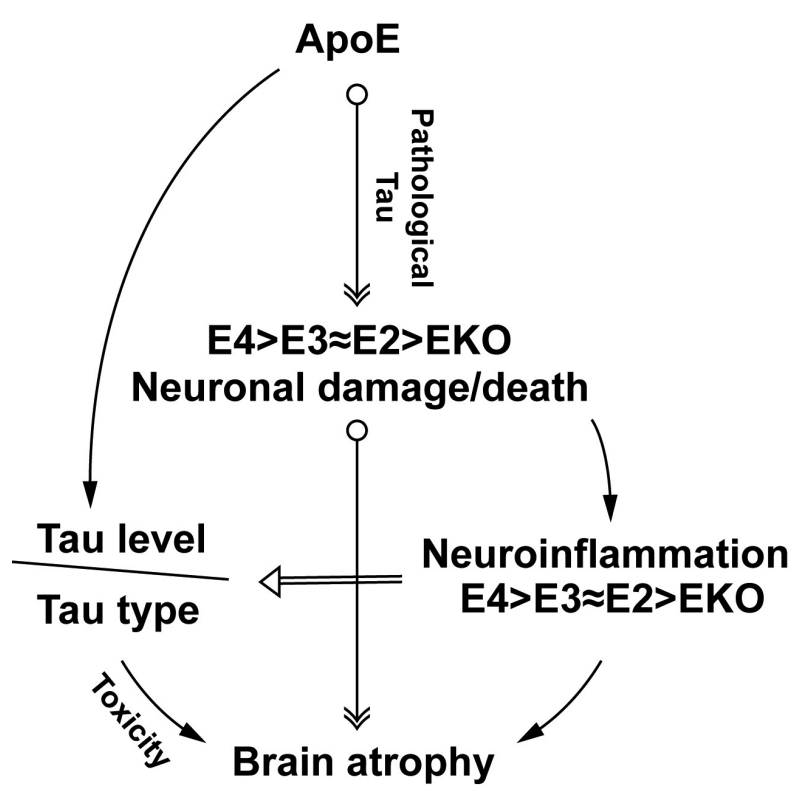

b ApoE<smiles>N#CO</smiles>

Tau level

Tau type

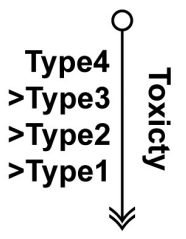

$E 4>E 3 \approx E 2>E K O$ neuronal death

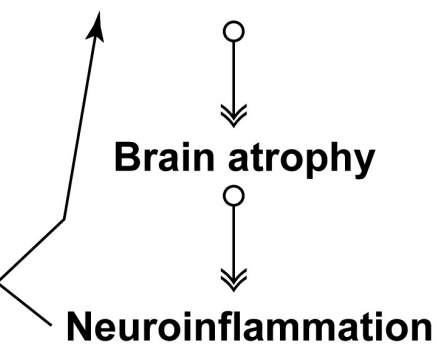

C<smiles></smiles>

Tau level

Tau type

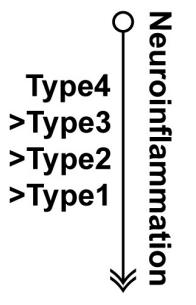

$E 4>E 3 \approx E 2>E K O$ Neuroinflammation<smiles>O[14CH2]O</smiles>

Neuronal death<smiles>O[14CH2]O</smiles>

Brain atrophy
Extended Data Figure 9 | Schematic summary of hypotheses. a, ApoE is essential for neuronal death under pathological conditions. With pathological tau accumulation, the presence of ApoE, especially ApoE4, renders the neurons more susceptible to degeneration, whereas the absence of ApoE protects neurons from death, resulting in neurodegeneration $(\mathrm{E} 4>\mathrm{E} 3 \approx \mathrm{E} 2>>\mathrm{EKO})$. Degenerating neurons further induce neuroinflammation, which is augmented by ApoE4 owing to its inherent higher innate immune reactivity, thereby exacerbating neurodegeneration further. Neuroinflammation may concomitantly affect tau pathology $y^{13}$, resulting in various $\mathrm{p}$-tau staining types that could also contribute to neurodegeneration. $\mathbf{b}$, ApoE affects tau pathogenesis, resulting in different $\mathrm{p}$-tau patterns, which may possess distinct neurotoxicity (type $4>$ type $3 \approx$ type $2>$ type 1 ), leading to different levels of neuronal death and brain atrophy $(\mathrm{E} 4>\mathrm{E} 3 \approx \mathrm{E} 2>>\mathrm{EKO})$. Neuroinflammation accompanying neurodegeneration will in turn exacerbate neuronal death. c, ApoE affects tau pathogenesis, resulting in different $\mathrm{p}$-tau patterns that may have different capacities to induce neuroinflammation (type $4>$ type $3 \approx$ type $2>$ type 1 ), which eventually leads to various degrees of neurodegeneration. 
Extended Data Table 1 | Possession of an $\varepsilon 4$ allele exacerbates regional neurodegeneration in human primary tauopathies

$$
\text { N Sex }
$$

$\begin{array}{lll}\text { Sex } & \text { Mean SD } \\ \text { (M) Age } & \end{array}$

Mean SD

Mean

SD

Mean SD
ND

D Mean

SD Mean

SD Mean SD

TDP-

Duration

Score

Score

$A \beta$
Score

43

Score

Onset Death

\begin{tabular}{|c|c|c|c|c|c|c|c|c|c|c|c|c|c|c|c|c|c|c|}
\hline CBD & 29 & 15 & 61 & 7.1 & 69 & 6.4 & 7.2 & 3.4 & 0.74 & 1.1 & 7.77 & 4.3 & 0.6 & 1.4 & 0.28 & 1 & 0.12 & 0.6 \\
\hline E4 & 6 & 4 & 60 & 4.2 & 67 & 5.7 & 6.5 & 2.1 & 0.76 & 1.1 & 7.75 & 4.6 & 1.77 & 2.1 & 0.55 & 1.4 & 0 & 0 \\
\hline no E4 & 23 & 11 & 62 & 7.7 & 69 & 6.6 & 7.3 & 3.6 & 0.73 & 1.1 & 7.78 & 4.2 & 0.32 & 0.9 & 0.2 & 0.8 & 0.16 & 0.7 \\
\hline Pick's & 17 & 8 & 59 & 6.3 & 68 & 6.5 & 9.6 & 2.6 & 1.07 & 1.6 & 6.08 & 4.5 & 0.69 & 1.6 & 0.01 & 0.1 & 0.1 & 0.6 \\
\hline E4 & 5 & 2 & 55 & 6.4 & 65 & 6.2 & 10.2 & 2.9 & 1.09 & 1.6 & 5.88 & 4.1 & 1.2 & 1.7 & 0 & 0 & 0.18 & 0.7 \\
\hline no E4 & 12 & 6 & 60 & 5.9 & 70 & 6.4 & 9.4 & 2.6 & 1.06 & 1.7 & 6.16 & 4.6 & 0.51 & 1.6 & 0.01 & 0.1 & 0.07 & 0.6 \\
\hline PSP & 33 & 13 & 64 & 7.5 & 72 & 6.9 & 8.2 & 3.1 & 0.53 & 1 & 7.08 & 4 & 1.07 & 1.9 & 0.07 & 0.4 & 0.25 & 0.8 \\
\hline no E4 & 25 & 12 & 62 & 7.5 & 71 & 7.1 & 8.5 & 3.1 & 0.5 & 1 & 7.23 & 4.1 & 0.67 & 1.5 & 0.01 & 0.2 & 0.27 & 0.9 \\
\hline All Dx & 79 & 36 & 62 & 7.3 & 70 & 6.8 & 8.1 & 3.2 & 0.72 & 1.2 & 7.1 & 4.2 & 0.82 & 1.7 & 0.13 & 0.7 & 0.17 & 0.7 \\
\hline E4 & 19 & 7 & 62 & 7.7 & 70 & 7.1 & 7.7 & 3 & 0.79 & 1.2 & 6.77 & 4.1 & 1.84 & 2.1 & 0.27 & 0.9 & 0.11 & 0.6 \\
\hline no E4 & 60 & 29 & 62 & 7.2 & 70 & 6.7 & 8.25 & 3.3 & 0.7 & 1.2 & 7.2 & 4.3 & 0.5 & 1.3 & 0.08 & 0.5 & 0.19 & 0.8 \\
\hline
\end{tabular}

ND score: neurodegeneration score, determined by [(microvacuolation + astrogliosis) / 2) + neuronal loss]

\begin{tabular}{|l|c|}
\hline begion & Total times region is used in analysis \\
\hline 3rd nucleus & 28 \\
\hline Anterior cingulate cortex & 51 \\
\hline CA1/subiculum & 76 \\
\hline CA2 & 72 \\
\hline CA3-4 & 75 \\
\hline Dentate gyrus & 73 \\
\hline Dorsal raphe & 23 \\
\hline Entorhinal cortex & 76 \\
\hline Mid front gyrus & 15 \\
\hline Periaqueductal grey & 40 \\
\hline Substatntia nigra & 40 \\
\hline Tectum & 40 \\
\hline
\end{tabular}

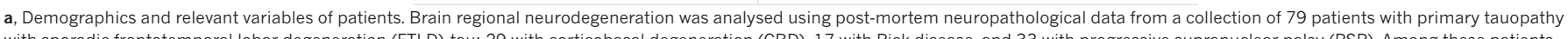

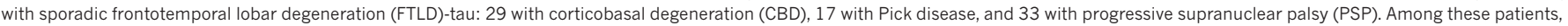

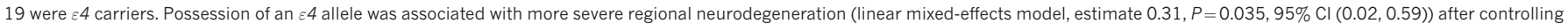

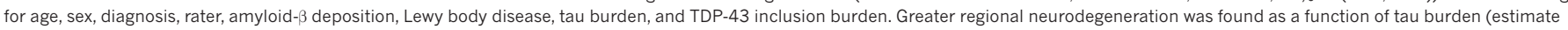
$0.17, P<0.001 .95 \% \mathrm{Cl}(0.14,0.20)$ ) and TDP-43 proteinopathy (estimate $0.22, P=0.003,95 \% \mathrm{Cl}(0.07,0.37)$ ), whereas amyloid- $\beta$ deposition was associated with less severe neurodegeneration (estimate $-0.08, P=0.044,95 \% \mathrm{Cl}(-0.15,-0.002)$ ). Lewy body disease, which was relatively uncommon in this cohort, showed no effect (estimate $-0.01, P=0.87,95 \% \mathrm{Cl}(-0.17,0.15)$ ). $\mathbf{b}, \mathrm{Brain}$ regions used for analysis. 


\section{nature research}

Corresponding author(s): David M. Holtzman

Initial submission $\bigotimes$ Revised version

Final submission

\section{Life Sciences Reporting Summary}

Nature Research wishes to improve the reproducibility of the work that we publish. This form is intended for publication with all accepted life science papers and provides structure for consistency and transparency in reporting. Every life science submission will use this form; some list items might not apply to an individual manuscript, but all fields must be completed for clarity.

For further information on the points included in this form, see Reporting Life Sciences Research. For further information on Nature Research policies, including our data availability policy, see Authors \& Referees and the Editorial Policy Checklist.

\section{- Experimental design}

1. Sample size

Describe how sample size was determined.

\section{Data exclusions}

Describe any data exclusions.

\section{Replication}

Describe whether the experimental findings were reliably reproduced.

\section{Randomization}

Describe how samples/organisms/participants were allocated into experimental groups.

\section{Blinding}

Describe whether the investigators were blinded to group allocation during data collection and/or analysis.
We utilized N 15 mice per group for most of the analysis, as this size gives us $80 \%$ power to see differences between groups of $>20 \%$ with two-sided significance of 0.05 in this P301S mouse model. We added more mice per group later to achieve a sample size of $\sim 20-30$ when we performed the statistical analysis for the $p$-tau staining pattern distribution, as this analysis requires larger sample size to achieve meaningful statistics. We also used this increased sample size for brain volume analysis in the revised manuscript.

For analysis of clinical disease progression in human AD patients, sample selection was done as follow: only participants that had an AD diagnosis at the last visit and had a CSF profile compatible with AD were included in our analyses. Non-AD dementia was excluded, as well as individuals in which the CDR at last assessment was equal to 0 . Individuals with diagnoses of neurological diseases other than AD were excluded. Not informative longitudinally measured CDR-SB was removed for each participant. After removing the non-informative data points only individuals with at least 1.5 year of follow-up were included. A total of 592 participants were included in the analyses.

All attempts at replication were successful

For biochemical assay and histological staining, the "RAND" function in EXCEL was applied to assign each sample a random number and sorted smallest to largest to generate random order. For gene expression assay, equal number of samples from each group were placed on each chip by complete randomization to avoid crosschip variability

Quantification of brain volume, AT8 staining, p-tau patterns, and fluorescent staining were performed by individuals blinded to samples IDs. Microglial/ astrocytic gene expression assays were performed by collaborators who were blinded to sample IDs. Cell culture images were taken and data quantified by individuals blinded to sample IDs. 
6. Statistical parameters

For all figures and tables that use statistical methods, confirm that the following items are present in relevant figure legends (or in the Methods section if additional space is needed).

$\mathrm{n} / \mathrm{a} \mid$ Confirmed

$\bigotimes$ The exact sample size $(n)$ for each experimental group/condition, given as a discrete number and unit of measurement (animals, litters, cultures, etc.)

$\square$ A description of how samples were collected, noting whether measurements were taken from distinct samples or whether the same

sample was measured repeatedly

$\bigotimes$ A statement indicating how many times each experiment was replicated

The statistical test(s) used and whether they are one- or two-sided (note: only common tests should be described solely by name; more complex techniques should be described in the Methods section)

$\bigotimes$ A description of any assumptions or corrections, such as an adjustment for multiple comparisons

$\bigotimes$ The test results (e.g. $P$ values) given as exact values whenever possible and with confidence intervals noted

$\square$ Х A clear description of statistics including central tendency (e.g. median, mean) and variation (e.g. standard deviation, interquartile range)

$\square$ Clearly defined error bars

See the web collection on statistics for biologists for further resources and guidance.

- Software

Policy information about availability of computer code

7. Software

Describe the software used to analyze the data in this study.

GraphPad Prism version 6.00 for Windows (GraphPad Software, La Jolla, CA) was used for all mouse and cell culture data analysis except for the p-tau staining pattern analysis, $R$ version 3.4.0 (The R Foundation for Statistical Computing) was used for statistical analysis. For human AD data, R version 3.3.3 "Another Canoe" platform x86_64-redhat-linux-gnu(64-bit) and the package nlme were used for linear mixed model. For human primary tauopathy data,

For manuscripts utilizing custom algorithms or software that are central to the paper but not yet described in the published literature, software must be made available to editors and reviewers upon request. We strongly encourage code deposition in a community repository (e.g. GitHub). Nature Methods guidance for providing algorithms and software for publication provides further information on this topic.

\section{- Materials and reagents}

Policy information about availability of materials

\section{Materials availability}

Indicate whether there are restrictions on availability of unique materials or if these materials are only available for distribution by a for-profit company.
All reagents including antibodies produced in house are available after completing an MTA from Washington University. APOE knockin mice may be purchased from Taconic. The Tau- 5 antibody can be obtained with an MTA from Michigan State, the institution that is now maintaining that antibody. 
9. Antibodies

Describe the antibodies used and how they were validated for use in the system under study (i.e. assay and species).
10. Eukaryotic cell lines

a. State the source of each eukaryotic cell line used.

b. Describe the method of cell line authentication used.

c. Report whether the cell lines were tested for mycoplasma contamination.

d. If any of the cell lines used are listed in the database of commonly misidentified cell lines maintained by ICLAC, provide a scientific rationale for their use.
AT8B: Thermo Fisher Scientific, MN1020B, validated for IHC, ICC, IF, ELISA, WB in human

CD68: AbD SeroTec, MCA1957, validated for IHC, IF, FC, WB, IP in mouse GFAP: EMD Millipore, MAB3402, clone GA5, Lot: 2724317, validated for ICC, IHC, $I H(P)$, and WB in Human, Rat, Mouse, Porcine, Chicken, Bovine, and Rabbit ApoE for western: Abcam, ab24139, validated for Dot blot, ELISA, IHC-Fr, IHC-P, and ICC/IF in human

ApoE for ELISA: HJ15.6, HJ15.4B, in house antibody, validated for ELISA, WB and IHC-Fr in human

a tubulin: Sigma, T5168, clone B-5-1-2, validated for indirect IF, WB, and radioimmunoassay in human, Chlamydomonas, African green monkey, chicken, mouse, bovine, rat, kangaroo rat, and sea urchin

Tau5: Gift from L. Binder, Northwestern University, Evanston, IL

HT7B: Thermo Fisher Scientific, MN1000B, validated for ELISA, IHC, and WB in Bovine and Human

GAPDH: Abcam, ab9484, Lot: GR174666-6, validated for WB, IHC-P, and FC in Mouse, Rat, Rabbit, Chicken, Cow, Dog, Human, Pig, Xenopus laevis, Cynomolgus monkey, and Chinese hamster

MAP2 :Thermo Fisher Scientific, OSM00030W, validated for WB and IHC in mouse and rat

N/A

N/A

N/A

N/A

\section{- Animals and human research participants}

Policy information about studies involving animals; when reporting animal research, follow the ARRIVE guidelines

\section{Description of research animals}

Provide details on animals and/or animal-derived materials used in the study.
Human ApoE2, ApoE3, and ApoE4 knockin (KI) mice (C57BL/6) were provided by Dr. Patrick M. Sullivan (Duke University) and ApoE knockout (KO) mice (C57BL/6) were purchased from The Jackson Laboratory (\#002052). P301S tau transgenic mice (The Jackson Laboratory, \#008169) on a B6/C3 background expressing human P301S 1N4R tau driven by PrP promoter were crossed to human ApoE KI mice to generate P301S hE/hE mice for all three ApoE isoforms, which were then crossed to ApoE KO mice to generate P301S hE/- mice. Separately, ApoE KI mice were crossed to ApoE KO mice to generate $\mathrm{hE} /-$ mice. P301S hE/- mice were then crossed with hE/- mice to generate P301S hE/hE, P301S hE/- and P301S -/littermates for all three ApoE isoforms. All tau transgenic mice for final analysis were kept at the same generation and had $\sim 90 \% \mathrm{C} 57 \mathrm{BL} / 6$ background by congenic test. Only male ApoE homozygote and knockout mice were used for analysis. WT mice were purchased from Charles River Laboratories (\#027) on a C57BL/6 background and were bred separately from the P301S/ApoE mice.

For cell culture experiments, primary astrocytes and microglia are derived from ApoE2KI, ApoE3KI, ApoE4KI, and APOEKO mice described above. Primary neurons are derived from CD-1 ${ }^{\circledR}$ IGS WT Mouse from Charles River (\#022).

Policy information about studies involving human research participants

\section{Description of human research participants}

Describe the covariate-relevant population characteristics of the human research participants.
This information can be found in Extended Data Table 8a for human primary tauopathies. 